\title{
Experimental Research On the Performance of a Novel Geo-Filament Anchor For An Earthen Architectural Site
}

Wang Yulan ( $\square$ ylwhbp@xauat.edu.cn )

Xi'an University of Architecture and Technology https://orcid.org/0000-0003-3097-4861

\section{Guo Jian}

Xi'an University of Architecture and Technology Architecture College

\section{Zhang Weixi}

Xi'an University of Architecture and Technology

\section{Lei Fan}

Shaanxi Provincial Institute of Cultural Heritage Xi'an China

\section{Research article}

Keywords: Earthen Architectural sites, Small sliders, Geotechnical Filament Anchor (GFA), In-situ experiments, Anchoring performance

Posted Date: November 1st, 2021

DOl: https://doi.org/10.21203/rs.3.rs-951337/v1

License: (a) (i) This work is licensed under a Creative Commons Attribution 4.0 International License. Read Full License 


\section{Abstract}

An efficient anchoring method, explicitly developed for small sliders, has hitherto been missing in the practice of earthen architecture conservation. Furthermore, anchoring performance studies conducted so far, have failed to fully take into account the soil characteristics of certain targets. To address these concerns, the conservation project conceived for the Gaochang Ruins, Turpan, in China, was selected as the testing ground to design a novel Geotechnical Filament Anchor (GFA) for reinforcing small sliders in the earthen historical ramparts. In-situ experiments were conducted for evaluating six parameters-anchoring length (L), GF thickness $(H)$, bore diameter $(D)$, grouting strength $(S)$, GFA surface status $(R)$, and inclination angle $(A)$. These parameters were varied in order to determine the effect they produce on anchoring performance, as demonstrated by the indicators, including tensile strength, destruction mode, load displacement ( $P-S)$ relation, and strain ( $\zeta-L)$ distribution characteristics of the novel GFA. Data acquired from the experiments, in combination with the conservation specifics of earthen architectural sites, anchoring performance, and safety reserve, were further employed to introduce a calculation formula for computing the designed force value $(N)$ through L. A simplified model depicting the shear stress distribution of the anchoring system under $N$ was devised by extracting the strain distribution data with respect to the GF-grouting interface. Taking into account the soil properties of the above-mentioned site, the shear stress diffusion coefficient (a) was conceptualized, the formula for the shear strength of the grouting material was devised, and the tolerable ranges of $L, D, H, R$, and $S$ were determined. Thus, a feasible anchoring method for small sliders used in earthen architectural sites is proposed, and validated by strong and reliable experimental and theoretical groundwork.

\section{Introduction}

Earthen architectural sites remain one of the most treasured cultural legacies for the whole of humankind. Keeping in mind the diversities present in the construction materials, vulnerability, structural complexity, and varied classifications of earthen architectural sites, cultural relics protection entities, constantly face the challenge of safeguarding and maintaining these historical remnants ${ }^{[1]}$.

In the present scenario, the issue of structural instability of these earth relics is predominantly resolved by adopting protection measures such as reinforced supporting and anchoring techniques. Anchoring technology finds extensive application in the domain of geotechnical engineering. Utilizing basic reinforcement equipment such as steel bars, steel strands, glass fiber reinforced polymer/plastic (GFRP), and aramid fiber reinforced polymer (AFRP) as the bolting material, a substantial number of laboratory and field tests have been conducted to study anchoring performance, interface mechanics theory, anchoring parameters, and so on ${ }^{[2-8]}$.

Nevertheless, the process of structural reinforcement for a particular earthen architectural site possesses certain characteristics, and it is not viable to copy and implement the reinforcement technique and the materials employed in a specific site in other sites as well. Therefore, new and different sorts of anchor rods have been developed and utilized for the structural reinforcement of diverse ruins. ${ }^{[9-11]}$ The anchored 
objects utilized mostly appear to be large sliders, and the rods are typically made of bamboo, wood, steel bars $^{[12-15]}$.

Majority of the related studies primarily emphasize on the impact generated by the bolt length, grouting material, and several other strengthening objects on the anchoring force and the stress distribution characteristics projected by the anchor rod-grouting interface ${ }^{[16-24]}$. However, minimal studies exist on the demand for anchoring performance with respect to the soil characteristics of these reinforced cultural relics, while no customized anchoring measures, whatsoever, have been developed for small sliders.

This paper has been framed around the research backdrop of the protection of small sliders in the Gaochang Ruins. An innovative Geotechnical Filament Anchor (GFA) was designed keeping in mind the soil properties of this site. The influence produced by each of the factors-anchoring length L, GF thickness $H$, bore diameter $D$, grouting material $S, G F$ surface state $R$, and inclination angle $A$, on the anchoring performance was meticulously evaluated. Based on the test phenomena and the associated mechanical analysis, the anchoring characteristics of this new GFA were summarized, providing the design and construction basis required for the anchoring of small sliders in such earthen architectural sites.

\section{Research Background}

Gaochang city, a significant site along the original Silk Road, was built in the first century BCE (mid-Western Han Dynasty) and was used as a garrison city for stationing troops of the Western Han dynasty in the western regions. A prolonged war led to the abandonment of the entire city at the end of the 13th century $C E$. The city was built more than 2,000 years ago and was used as a key transportation hub for more than 1,300 years.

The plane layout of the Gaochang Ruins appears to be an irregular square, which is divided into three parts: an outer city, an inner city, and a palace city (Fig. 1). The circumference of the outer city fortifications is about 5.5 kilometers, covering an area of 198 hectares, and is categorized under the early architectural sites group in Xinjiang of China and represents the largest preserved area among such sites. Most of these ramparts constitute of rammed loess, which are 6 11 meters high and about 10 meters thick, while the rammed layer seems to be about 8 10 centimeters thick.

The various natural and man-made destructive events inflicted over thousands of years have deteriorated a large portion of these rampart relics, with most of the existing ramparts exhibiting varying degrees of hidden structural damage (Fig. 2, Fig. 3). To resolve the above-mentioned issues, practical engineering grouting, adobe support, bamboo, and wood anchor reinforcement techniques were adopted (Fig. 4). Bamboo and wood anchor bolts possess the advantages of enhanced control for structural displacement and conform with the principle of cultural relic protection; however, attaining adequate hole size as well as depth are necessary to achieve optimum anchoring performance. In case of the reinforcement of small sliders, certain drawbacks are present such as increased damage and degeneration of the anchoring performance.

Based on the above-mentioned problems, a new type of GFA was designed to efficiently reinforce the small sliders for the protection of the Gaochang Ruins, followed by the experimentation and evaluation of its 


\section{Experiment Scheme}

The novel geo-filament (GF) utilized in this study is a high-fiber synthetic material, signifying a ribbon material formed by the process of extrusion (Fig. 5). The material property test demonstrates that the tensile strength of this GF seems to be particularly high, and its physical and mechanical parameters are as shown in Table 1.

Table 1

GF material performance

\begin{tabular}{|llll|}
\hline $\begin{array}{l}\text { Ultimate tension } \\
\boldsymbol{k N}\end{array}$ & $\begin{array}{l}\text { Tensile strength } \\
\mathrm{MPa}\end{array}$ & $\begin{array}{l}\text { Elongation } \\
\%\end{array}$ & $\begin{array}{l}\text { Friction coefficient } \\
\boldsymbol{f}\end{array}$ \\
\hline 20.5 & 273 & 1.4 & 0.6 \\
\hline
\end{tabular}

\subsection{Experiment design and specimen preparation:}

A total of 6 independent experimental sets were designed, and 19 experiment teams were employed with 3 experimental parts assigned to each team. Six independent experimental sets were then divided as follows: anchor rod length L (5 teams), geo-filament thickness $H$ (3 teams), bore diameter D (3 teams), grouting strength $S$ (3 teams), surface state $R$ of anchor rod (3 teams), and deployment angle $A$ of anchor rod (3 teams). The design parameters of the test parts are as shown in Table 2. The single-factor test method was adopted, where the effect of a single factor on the anchoring force at a given time is evaluated under similar environmental conditions, keeping the other five factors as constant.

The GF was cut in a length as required by the test, while $400 \mathrm{~mm}$ of free section was reserved for anchoring with the pull-off tester. The outer side of the GF was evenly wound once with hemp rope, the spacing was identified in the experiment scheme, while the end part was wound three times and was then uniformly coated with epoxy resin. It is vital to ensure that the epoxy resin impregnates the hemp rope properly during coating, to closely fuse it with the geo-filament.

To assess the stress distribution of the geo-filament and grouting interface, the geo-filament anchor was divided into four equidistant parts. The strain foil of the test anchor rod is required to be deployed at the two ends, 1/4 position, 1/2 position, and 3/4 position. The compensator needs to be deployed around the middle position. The schematic diagram of anchor production is shown in Figure 6 . The diagram of the cross section of the anchor is shown in Figure 7. Finally, epoxy resin and waterproof tape were used as coatings for protection. The lead and shielded wires were welded and numbered in sequence, as shown in Figure 8. 
Table 2

Specimen grouping design parameter table

\begin{tabular}{|c|c|c|c|c|c|c|c|}
\hline $\begin{array}{l}\text { Parameter } \\
\text { Factor }\end{array}$ & $\begin{array}{l}\text { Anchor } \\
\text { length } \\
\text { (mm) }\end{array}$ & $\begin{array}{l}\text { thickness } \\
\text { (piece) }\end{array}$ & $\begin{array}{l}\text { Bore } \\
\text { diameter } \\
(\mathrm{mm})\end{array}$ & $\begin{array}{l}\text { Grouting } \\
\text { number }\end{array}$ & $\begin{array}{l}\text { Ribbed } \\
\text { spacing } \\
(\mathrm{mm})\end{array}$ & $\begin{array}{l}\text { Angel of } \\
\text { inclination }\left({ }^{\circ}\right)\end{array}$ & $\begin{array}{l}\text { Numbel } \\
\text { of test } \\
\text { pieces }\end{array}$ \\
\hline \multirow{5}{*}{$\begin{array}{l}\text { L Series } \\
\text { (Anchor } \\
\text { length) }\end{array}$} & $800(L 8)$ & \multirow[t]{5}{*}{ Single } & \multirow[t]{5}{*}{45} & \multirow[t]{5}{*}{$S 2$} & \multirow[t]{5}{*}{30} & \multirow[t]{5}{*}{$0^{\circ}$} & 3 \\
\hline & $1200(L 12)$ & & & & & & 3 \\
\hline & $1500(L 15)$ & & & & & & 3 \\
\hline & $2000(L 20)$ & & & & & & 3 \\
\hline & $3000(L 30)$ & & & & & & 3 \\
\hline \multirow{2}{*}{$\begin{array}{l}\text { H Series } \\
\text { (One- } \\
\text { sided } \\
\text { thickness) }\end{array}$} & \multirow[t]{2}{*}{1200} & Single(H1) & \multirow[t]{2}{*}{45} & \multirow[t]{2}{*}{ S2 } & \multirow[t]{2}{*}{30} & \multirow[t]{2}{*}{$0^{\circ}$} & 3 \\
\hline & & $\begin{array}{l}\text { Double } \\
\operatorname{root}(H 2)\end{array}$ & & & & & 3 \\
\hline \multirow{3}{*}{$\begin{array}{l}\text { D Series } \\
\text { (Aperture) }\end{array}$} & \multirow[t]{3}{*}{1200} & \multirow[t]{3}{*}{ Single } & $45(D 45)$ & \multirow[t]{3}{*}{ S2 } & \multirow[t]{3}{*}{30} & \multirow[t]{3}{*}{$0^{\circ}$} & 3 \\
\hline & & & $75(D 75)$ & & & & 3 \\
\hline & & & $80(D 80)$ & & & & 3 \\
\hline \multirow{3}{*}{$\begin{array}{l}\text { S Series } \\
\text { (Grouting } \\
\text { strength) }\end{array}$} & \multirow[t]{3}{*}{1200} & \multirow[t]{3}{*}{ Single } & \multirow[t]{3}{*}{45} & $S 1$ & \multirow[t]{3}{*}{30} & \multirow[t]{3}{*}{$0^{\circ}$} & 3 \\
\hline & & & & $S 2$ & & & 3 \\
\hline & & & & s3 & & & 3 \\
\hline \multirow{3}{*}{$\begin{array}{l}\text { R Series } \\
\text { (Ribbed } \\
\text { spacing) }\end{array}$} & \multirow[t]{3}{*}{1200} & \multirow[t]{3}{*}{ Single } & \multirow[t]{3}{*}{45} & \multirow[t]{3}{*}{$S 2$} & $O(R O)$ & \multirow[t]{3}{*}{$0^{\circ}$} & 3 \\
\hline & & & & & $30(R 30)$ & & 3 \\
\hline & & & & & $100(R 100)$ & & 3 \\
\hline \multirow{3}{*}{$\begin{array}{l}\text { A Series } \\
\text { (slope) }\end{array}$} & \multirow[t]{3}{*}{1200} & \multirow[t]{3}{*}{ Single } & \multirow[t]{3}{*}{45} & \multirow[t]{3}{*}{ S2 } & 30 & $0^{\circ}(A O)$ & 3 \\
\hline & & & & & & $10^{\circ}(A 10)$ & 3 \\
\hline & & & & & & $15^{\circ}(A 15)$ & 3 \\
\hline
\end{tabular}

\subsection{Material characteristics of the experiment:}

(1) Mechanical properties of the rammed earth:

The rampart relics of Gaochang are primarily made of rammed earth. To reduce the experimental deviation, the core samples were drilled in the local test area, to examine the material parameters shown in Table 3. 
Table 3

Physical and mechanical parameters of rammed earth

\begin{tabular}{|lllllll|}
\hline $\begin{array}{l}\text { Test } \\
\text { lock }\end{array}$ & $\begin{array}{l}\text { Moisture } \\
\text { content } \omega \\
\text { /\% }\end{array}$ & $\begin{array}{l}\text { Density } \\
\boldsymbol{\rho}\end{array}$ & $\begin{array}{l}\text { Angle of } \\
\text { internal } \\
\text { friction } \boldsymbol{l} /(\boldsymbol{O})\end{array}$ & $\begin{array}{l}\text { Cohesion } \\
\text { strength/kN }\end{array}$ & $\begin{array}{l}\text { Test block } \\
\text { compressive } \\
\text { strength (MPa) }\end{array}$ & $\begin{array}{l}\text { Compression } \\
\text { Elastic } \\
\text { Modulus } \\
\text { E/MPa }\end{array}$ \\
\hline 1 & 3.2 & 1.89 & 25.5 & 28.5 & 1.61 & 180.32 \\
\hline 2 & 2.8 & 1.76 & 23.6 & 27.6 & 1.55 & 179.40 \\
\hline 3 & 2.5 & 1.81 & 24.4 & 28.2 & 1.73 & 188.80 \\
\hline Mean & 2.8 & 1.82 & 24.5 & 28.1 & 1.63 & 182.84 \\
\hline
\end{tabular}

(2) Mechanical properties of the grouting body:

The grouting bodies were quantified into three proportions, in accordance to the factual engineering experiences $(S 1, S 2, S 3)$. The grouting body proportioning was calculated by the weight proportioning.

S1 grouting body proportioning: earth: cement: fly ash = 90:5:5. 5\% ludox emulsion is mixed.

S2 grouting body proportioning: earth: cement: fly ash $=85: 5: 10.5 \%$ sweller is used.

S3 grouting body proportioning: earth: cement: fly ash $=80: 10: 10.5 \%$ sweller emulsion is mixed.

The ratio of water to ash for the three slurries was $31 \%$ (weight ratio). The $70.7 \times 70.7 \mathrm{~mm}$ mortar test die was utilized to make the grouting test block. After a 34-day maintenance, its compressive mechanical performance is shown in Table 4.

Table4 Mechanical properties of grout 


\begin{tabular}{|c|c|c|c|c|c|}
\hline \multirow[t]{2}{*}{ Test block } & \multirow[t]{2}{*}{ Number } & \multicolumn{2}{|c|}{ Compressive Strength (MPa) } & \multicolumn{2}{|c|}{$\begin{array}{l}\text { Compression Elastic Modulus } \\
10^{5}(\mathrm{MPa})\end{array}$} \\
\hline & & Experimental value & average & Experimental value & average \\
\hline \multirow[t]{3}{*}{ No. 1 grout } & 1 & 1.94 & \multirow[t]{3}{*}{1.93} & 65.65 & \multirow[t]{3}{*}{64.89} \\
\hline & 2 & 1.86 & & 63.26 & \\
\hline & 3 & 1.98 & & 65.78 & \\
\hline \multirow[t]{3}{*}{ No. 2 grout } & 1 & 2.81 & \multirow[t]{3}{*}{2.81} & 68.75 & \multirow[t]{3}{*}{68.60} \\
\hline & 2 & 2.72 & & 67.38 & \\
\hline & 3 & 2.91 & & 69.68 & \\
\hline \multirow[t]{3}{*}{ No. 3 grout } & 1 & 5.64 & \multirow[t]{3}{*}{5.41} & 188.5 & \multirow[t]{3}{*}{184.06} \\
\hline & 2 & 5.32 & & 182.3 & \\
\hline & 3 & 5.27 & & 181.4 & \\
\hline
\end{tabular}

\section{In-situ Test}

The east section of the north rampart in the Gaochang Ruins was selected as the experimental section and the pull-out test of geo-filament bolts were conducted on the test site itself. The soil conditions appeared to be similar to those of the reinforcing objects.

\subsection{Installation of the test parts:}

The vertical and horizontal spacing between the borehole was set to be more than $1 \mathrm{~m}$, to avoid mutual influences. The bore diameter was cleaned by using an electric blower's peripheral brush and moisturized with a soft brush and a small sprinkler. The field anchoring test parts are shown in Figure 9.

\subsection{Test method:}

The TDS-303 data collection instrument from Japan was employed as the strain collector (Fig. 10a), with a measurement range of -20000冈20000. The KFG general strain foil was selected. The sensitive grating was $3 \times 10 \mathrm{~mm}$ (the resistance was $120.8 \pm 0.1$ and sensitivity was $2.14 \pm 1 \%$ ). The anchor rod tension meter's measurement range was $0-100 \mathrm{kN}$ and the associated pulling force stroke was $150 \mathrm{~mm}$. To match the tensile strength tester of the anchor rod, a steel plate was utilized to build the reaction frame. A wood block was employed for padding between the reaction frame and the relics (Fig. 10b). A shift sensor was installed on the steel reaction frame. The experimental device is shown in Figure 11.

The experiment was conducted in the month of May, at the east section of the north rampart in Gaochang, Turpan, Xinjiang. On account of the experiment site and the weather factor, the in-situ drawing test was performed for the embedded anchor rod after the grouting body has solidified completely (about 40 days). 
$200 \mathrm{~N}$ pulling force was first applied prior to the loading, to eliminate any gap between the devices. Subsequently, $50 \mathrm{~N}$ force was applied per second for the rest of the experiment until the test part was destroyed ${ }^{[18,19]}$.

\section{Analysis Of The Test Results 5.1 Analysis of the anchoring performance:}

The experimental data was arranged and observed, and the experimental phenomena was recorded, followed by acquisition of the drawing destruction mode and maximal anchoring force in different experimental groups, shown in Table 5-10 (The data marked as " $*$ " is not considered).

Table 5 The test results by different anchor rod length. 


\begin{tabular}{|c|c|c|c|}
\hline Number & Destruction mode $\square k N \square$ & $\begin{array}{l}\text { Fracture } \\
\text { load } \square k N \square\end{array}$ & Average $\llbracket k N \square$ \\
\hline$L 8-1$ & $\begin{array}{l}\text { GF deformation } \square 2.3 \square \rightarrow \text { grouting cracking } \square 6.8 \square \rightarrow G F \text { pull out } \\
\square 7.8 \square\end{array}$ & 7.8 & \multirow[t]{3}{*}{7.95} \\
\hline$L 8-2$ & $\begin{array}{l}\text { GF deformation } \square 1.6 \square \rightarrow \text { grouting cracking } \square 5.2 \square \rightarrow \text { GF pull out } \\
\square 5.4 \square\end{array}$ & $5.4^{*}$ & \\
\hline$L 8-3$ & $\begin{array}{l}\text { GF deformation } \square 2.2 \square \rightarrow \text { grouting cracking } \square 7.3 \square \rightarrow G F \text { pull out } \\
\square 8.1 \square\end{array}$ & 8.1 & \\
\hline$L 12-1$ & $\begin{array}{l}\text { GF deformation } \square 2.3 \square \rightarrow \text { grouting cracking } \square 7.6 \square \rightarrow G F \text { pull out } \\
\square 11.5 \square\end{array}$ & 11.5 & \multirow[t]{3}{*}{10.63} \\
\hline$L 12-2$ & $\begin{array}{l}\text { GF deformation } \square 2.4 \square \rightarrow \text { grouting cracking } \square 7.8 \square \rightarrow G F \text { pull out } \\
\square 9.8 \square\end{array}$ & 9.8 & \\
\hline$L 12-3$ & $\begin{array}{l}\text { GF deformation } \square 2.0 \square \rightarrow \text { grouting cracking } \square 8.1 \square \rightarrow \text { GF pull out } \\
\square 10.6 \square\end{array}$ & 10.6 & \\
\hline$L 15-1$ & $\begin{array}{l}\text { GF deformation } \square 2.3 \square \rightarrow \text { grouting cracking } \square 6.5 \square \rightarrow \text { grouting } \\
\text { crushing } \square 11.2 \square \rightarrow \text { GF pull out } \square 15.5 \square\end{array}$ & 15.5 & \multirow[t]{3}{*}{16.47} \\
\hline$L 15-2$ & $\begin{array}{l}\text { GF deformation } \square 2.5 \square \rightarrow \text { grouting cracking } \square 7.1 \square \rightarrow \text { grouting } \\
\text { crushing } \square 13.6 \square \rightarrow G F \text { pull out } \square 17.1 \square\end{array}$ & 17.1 & \\
\hline$L 15-3$ & $\begin{array}{l}\text { GF deformation } \square 2.3 \square \rightarrow \text { grouting cracking } \square 7.5 \square \rightarrow \text { grouting } \\
\text { crushing } \square 12.8 \square \rightarrow \text { GF pull out } \square 16.8 \square\end{array}$ & 16.8 & \\
\hline$L 20-1$ & $\begin{array}{l}\text { GF deformation } \square 2.3 \square \rightarrow \text { grouting cracking } \square 7.5 \square \rightarrow \text { grouting } \\
\text { crushing } \square 12.3 \square \rightarrow \text { GF pull out } \square 18.7 \square\end{array}$ & 18.7 & \multirow[t]{3}{*}{18.55} \\
\hline$L 20-2$ & $\begin{array}{l}\text { GF deformation } \square 2.8 \square \rightarrow \text { grouting cracking } \square 8.2 \square \rightarrow \text { grouting } \\
\text { crushing } \square 15.1 \square \rightarrow \text { GF pull out } \square 15.6 \square\end{array}$ & $15.6^{*}$ & \\
\hline$L 20-3$ & $\begin{array}{l}\text { GF deformation } \square 2.3 \square \rightarrow \text { grouting cracking } \square 7.3 \square \rightarrow \text { grouting } \\
\text { crushing } \square 11.8 \square \rightarrow G F \text { pull out } \square 18.4 \square\end{array}$ & 18.4 & \\
\hline L30-1 & $\begin{array}{l}\text { GF deformation } \square 2.6 \square \rightarrow \text { grouting cracking } \square 7.8 \square \rightarrow \text { grouting } \\
\text { crushing } \square 11.5 \square \rightarrow G F \text { fracture } \square 19.8 \square\end{array}$ & 19.8 & \multirow[t]{3}{*}{19.83} \\
\hline L30-2 & $\begin{array}{l}\text { GF deformation } \square 2.4 \square \rightarrow \text { grouting cracking } \square 7.5 \square \rightarrow \text { grouting } \\
\text { crushing } \square 13.2 \square \rightarrow \text { GF fracture } \square 19.6 \square\end{array}$ & 19.6 & \\
\hline L30-3 & $\begin{array}{l}\text { GF deformation } \square 2.2 \square \rightarrow \text { grouting cracking } \square 8.2 \square \rightarrow \text { grouting } \\
\text { crushing } \square 12.8 \square \rightarrow G F \text { fracture } \square 20.1 \square\end{array}$ & 20.1 & \\
\hline
\end{tabular}

Table 6 Test results of different anchor rod diameter. 


\begin{tabular}{|c|c|c|c|}
\hline Number & Destruction mode $\llbracket k N \square$ & $\begin{array}{l}\text { Fracture } \\
\text { load } \square k N \square\end{array}$ & Average $\square k N \square$ \\
\hline$H 7-1$ & $\begin{array}{l}\text { GF deformation } \square 2.5 \square \rightarrow \text { grouting cracking } \square 8.0 \square \rightarrow \text { GF pull out } \\
\square 10.6 \square\end{array}$ & 10.6 & 11.16 \\
\hline$H 1-2$ & $\begin{array}{l}\text { GF deformation } \square 2.5 \square \rightarrow \text { grouting cracking } \square 8.2 \square \rightarrow G F \text { pull out } \\
\square 11.7 \square\end{array}$ & 11.7 & \\
\hline$H 1-3$ & $\begin{array}{l}\text { GF deformation } \square 2.2 \square \rightarrow \text { grouting cracking } \square 7.5 \square \rightarrow G F \text { pull out } \\
\square 11.2 \square\end{array}$ & 11.2 & \\
\hline$H 2-1$ & $\begin{array}{l}\text { GF deformation } \square 3.2 \square \rightarrow \text { grouting cracking } \square 7.5 \square \rightarrow \text { grouting } \\
\text { crushing } \square 12.3 \square \rightarrow \text { GF pull out } \square 14.2 \square\end{array}$ & 14.2 & 13.53 \\
\hline$H 2-2$ & $\begin{array}{l}\text { GF deformation } \square 3.5 \square \rightarrow \text { grouting cracking } \square 7.1 \square \rightarrow \text { grouting } \\
\text { crushing } \square 12.6 \square \rightarrow G F \text { pull out } \square 13.6 \square\end{array}$ & 13.6 & \\
\hline$H 2-3$ & $\begin{array}{l}\text { GF deformation } \square 3.5 \square \rightarrow \text { grouting cracking } \square 7.9 \square \rightarrow \text { grouting } \\
\text { crushing } \square 11.8 \square \rightarrow \text { GF pull out } 12.8 \square\end{array}$ & 12.8 & \\
\hline
\end{tabular}

Table 7 The test results by different bore diameter.

\begin{tabular}{|c|c|c|c|}
\hline Number & Destruction mode $\square k N \rrbracket$ & $\begin{array}{l}\text { Fracture load } \\
\square k N \square\end{array}$ & Average $\square \mathrm{kN} \square$ \\
\hline$D 45-1$ & $\begin{array}{l}\text { GF deformation } \square 2.3 \square \rightarrow \text { grouting cracking }[7.4 \square \\
\rightarrow \text { GF pull out } \square 11.5 \square\end{array}$ & 11.5 & \multirow[t]{3}{*}{10.77} \\
\hline$D 45-2$ & $\begin{array}{l}\text { GF deformation } \square 2.1 \square \rightarrow \text { grouting cracking } \square 7.8 \square \\
\rightarrow \text { GF pull out } \square 10.1 \square\end{array}$ & 10.1 & \\
\hline D45-3 & $\begin{array}{l}\text { GF deformation } \square 2.2 \square \rightarrow \text { grouting cracking } \square 7.5 \square \\
\rightarrow \text { GF pull out } \square 10.7 \square\end{array}$ & 10.7 & \\
\hline D75-1 & $\begin{array}{l}\text { GF deformation } \square 2.4 \square \rightarrow \text { grouting cracking } \square 6.3 \square \\
\rightarrow \text { GF pull out } \square 8.1 \square\end{array}$ & $8.1^{*}$ & \multirow[t]{3}{*}{10.5} \\
\hline D75-2 & $\begin{array}{l}\text { GF deformation } \square 2.5 \square \rightarrow \text { grouting cracking } \square 6.5 \square \\
\rightarrow \text { GF pull out } \square 10.7 \square\end{array}$ & 10.7 & \\
\hline$D 75-2$ & $\begin{array}{l}\text { GF deformation } \square 2.2 \square \rightarrow \text { grouting cracking } \square 6.6 \square \\
\rightarrow \text { GF pull out } \square 10.3 \square\end{array}$ & 10.3 & \\
\hline$D 80-1$ & GF deformation $\square 2.3 \square \rightarrow$ GF pull out $\square 7.6 \square$ & 7.6 & \multirow[t]{3}{*}{7.63} \\
\hline$D 80-2$ & GF deformation $\square 2.2 \square \rightarrow G F$ pull out $\square 8.2 \square$ & 8.2 & \\
\hline$D 80-3$ & GF deformation $\square 2.7 \square \rightarrow G F$ pull out $\square 7.4 \square$ & 7.4 & \\
\hline
\end{tabular}

Table 8 The test results by different grouting strength. 


\begin{tabular}{|c|c|c|c|}
\hline Number & Destruction mode $\square \mathrm{kN} \square$ & $\begin{array}{l}\text { Fracture load } \\
\square k N \square\end{array}$ & Average $\square k N \square$ \\
\hline$S 1-1$ & $\begin{array}{l}\text { GF deformation } \square 2.3 \square \rightarrow \text { grouting cracking } \square 4.5 \square \rightarrow \text { GF pull out } \\
\square 7.3 \square\end{array}$ & 7.3 & \multirow[t]{3}{*}{7.73} \\
\hline$S 1-2$ & $\begin{array}{l}\text { GF deformation } \square 2.5 \square \rightarrow \text { grouting cracking } \square 3.7 \square \rightarrow \text { GF pull out } \\
\square 7.9 \square\end{array}$ & 7.9 & \\
\hline$S 1-3$ & $\begin{array}{l}\text { GF deformation } \square 2.1 \square \rightarrow \text { grouting cracking } \square 4.3 \square \rightarrow \text { GF pull out } \\
\square 7.7 \square\end{array}$ & 7.7 & \\
\hline$S 2-1$ & $\begin{array}{l}\text { GF deformation } \square 2.1 \square \rightarrow \text { grouting cracking } \square 7.5 \square \rightarrow \text { GF pull out } \\
\square 11.4 \square\end{array}$ & 11.4 & \multirow[t]{3}{*}{11.43} \\
\hline$S 2-2$ & $\begin{array}{l}\text { GF deformation } \square 2.3 \square \rightarrow \text { grouting cracking } \square 8.2 \square \rightarrow \text { GF pull out } \\
\square 12.1 \square\end{array}$ & 12.1 & \\
\hline$S 2-3$ & $\begin{array}{l}\text { GF deformation } \square 1.7 \square \rightarrow \text { grouting cracking } \square 7.1 \square \rightarrow \text { GF pull out } \\
t \square 10.8 \square\end{array}$ & 10.8 & \\
\hline S3-1 & $\begin{array}{l}\text { GF deformation } \square 2.1 \square \rightarrow \text { soil-part loosening } \square 14.7 \square \rightarrow \text { grouting } \\
\text { pull out } \square 17.9 \square\end{array}$ & 17.9 & \multirow[t]{3}{*}{17.53} \\
\hline S3-2 & $\begin{array}{l}\text { GF deformation } \square 1.8 \square \rightarrow \text { soil-part loosening } \square 14.1 \square \rightarrow \text { grouting } \\
\text { pull out } 17.5 \square\end{array}$ & 17.5 & \\
\hline s3-3 & $\begin{array}{l}\text { GF deformation } \square 1.9 \square \rightarrow \text { soil-part loosening } \square 13.3 \square \rightarrow \text { grouting } \\
\text { pull out } 17.2 \square\end{array}$ & 17.2 & \\
\hline
\end{tabular}

Table 9 The test results by different surface state.

\begin{tabular}{|c|c|c|c|}
\hline Number & Destruction mode $\square k N \square$ & $\begin{array}{l}\text { Fracture load } \\
\square k N \square\end{array}$ & Average $\square k N \square$ \\
\hline$R 0-1$ & GF deformation $\square 2.1 \square \rightarrow$ GF pull out $\square 4.7 \square$ & 4.7 & \multirow[t]{3}{*}{4.33} \\
\hline$R O-2$ & GF deformation $\square 2.3 \square \rightarrow$ GF pull out $\square 4.5 \square$ & 4.5 & \\
\hline$R O-3$ & GF deformation $\square 2.0 \square \rightarrow$ GF pull out $\square 3.8 \square$ & 3.8 & \\
\hline$R 30-1$ & $\begin{array}{l}\text { GF deformation } \square 2.0 \square \rightarrow \text { grouting cracking } \square 7.5 \square \rightarrow G F \text { pull } \\
\text { out } \square 11.4 \square\end{array}$ & 11.4 & \multirow[t]{3}{*}{11.06} \\
\hline$R 30-2$ & $\begin{array}{l}\text { GF deformation } \square 2.4 \square \rightarrow \text { grouting cracking } \square 7.7 \square \rightarrow G F \text { pull } \\
\text { out } \square 10.6 \square\end{array}$ & 10.6 & \\
\hline$R 30-3$ & $\begin{array}{l}\text { GF deformation } \square 2.1 \square \rightarrow \text { grouting cracking } \square 7.2 \square \rightarrow \text { GF pull } \\
\text { out } \square 11.2 \square\end{array}$ & 11.2 & \\
\hline$R 100-1$ & GF deformation $\square 2.2 \square \rightarrow$ GF pull out $\square 7.3 \square$ & 7.3 & \multirow[t]{3}{*}{7.53} \\
\hline$R 100-2$ & GF deformation $\square 2.0 \square \rightarrow$ GF pull out $\square 7.5 \square$ & 7.5 & \\
\hline$R 100-3$ & GF deformation $\square 2.4 \square \rightarrow$ GF pull out $\square 7.8 \square$ & 7.8 & \\
\hline
\end{tabular}

Table 10 The test results by different deployment angle of anchor rod. 


\begin{tabular}{|c|c|c|c|}
\hline Number & Destruction mode $\square k N \square$ & $\begin{array}{l}\text { Fracture load } \\
\square k N \square\end{array}$ & Average $\square k N \square$ \\
\hline$A 0-1$ & $\begin{array}{l}\text { GF deformation } \square 2.0 \square \rightarrow \text { grouting cracking } \square 7.2 \square \rightarrow \text { GF pull } \\
\text { out } \square 10.3 \square\end{array}$ & 10.3 & \multirow[t]{3}{*}{10.77} \\
\hline$A 0-2$ & $\begin{array}{l}\text { GF deformation } \square 2.2 \square \rightarrow \text { grouting cracking } \square 8.1 \square \rightarrow G F \text { pull } \\
\text { out } \square 11.2 \square\end{array}$ & 11.2 & \\
\hline$A 0-3$ & $\begin{array}{l}\text { GF deformation } \square 2.3 \square \rightarrow \text { grouting cracking } \square 7.6 \square \rightarrow G F \text { pull } \\
\text { out } \square 10.8 \square\end{array}$ & 10.8 & \\
\hline A10-1 & $\begin{array}{l}\text { GF deformation } \square 1.8 \square \rightarrow \text { grouting cracking } \square 6.3 \square \rightarrow G F \text { pull } \\
\text { out } \square 9.4 \square\end{array}$ & 9.4 & \multirow[t]{3}{*}{8.97} \\
\hline A10-2 & $\begin{array}{l}\text { GF deformation } \square 2.0 \square \rightarrow \text { grouting cracking } \square 7.5 \square \rightarrow \text { GF pull } \\
\text { out } \square 10.2 \square\end{array}$ & 10.2 & \\
\hline A10-3 & $\begin{array}{l}\text { GF deformation } \square 2.1 \square \rightarrow \text { grouting cracking } \square 6.6 \square \rightarrow G F \text { pull } \\
\text { out } \square 7.3 \square\end{array}$ & 7.3 & \\
\hline$A 15-1$ & $\begin{array}{l}\text { GF deformation } \square 1.9 \square \rightarrow \text { grouting cracking } \square 4.7 \square \rightarrow \text { GF pull } \\
\text { out } \square 7.1 \square\end{array}$ & 7.1 & \multirow[t]{3}{*}{6.37} \\
\hline A15-2 & $\begin{array}{l}\text { GF deformation } \square 2.3 \square \rightarrow \text { grouting cracking } \square 5.1 \square \rightarrow G F \text { pull } \\
\text { out } \square 6.7 \square\end{array}$ & 6.7 & \\
\hline A15-3 & $\begin{array}{l}\text { GF deformation } \square 2.0 \square \rightarrow \text { grouting cracking } \square 4.6 \square \rightarrow G F \text { pull } \\
\text { out } \square 5.3 \square\end{array}$ & 5.3 & \\
\hline
\end{tabular}

As evident from the experimental results, the destruction mode does not occur in isolation. Several destruction modes transpire during the drawing process. When the anchorage system suffers a large displacement, it is considered as the final destruction mode. Four main anchorage system destruction modes can be obtained: geo-filament anchor pullout (Fig. 12a), grout-soil damage (Fig. 12b), geo-filament fracture (Fig. 12C), soil-part loosening and grouting pullout (Fig. 12d).

By summarizing the destruction phenomena and the properties of each destruction stage, the following conclusions can be drawn:

a. The filament-grout interface damage emerges to be the primary destruction mode of the anchorage system. Only with the robust strength level of the grouting can the grout-soil interface damage be triggered; there exists complete exertion of the anchoring system force at this moment. Hence, the grout-soil interface damage appears to be an ideal destruction mode, However, it can lead to the destruction of the site itself, hence, such a destruction mode appears non-conducive for heritage protection.

b. When the anchoring length reaches the depth of about $3000 \mathrm{~mm}$, the maximal anchoring force seems to be almost equal to the geo-filament's breaking tension value, thus resulting in the geo-filament fracture. c. The anchoring length $(L)$, grouting strength $(S)$, and surface state $(R)$ are factors sensitive to the enhancement of the anchoring performance of the geo-filament bolt, while the geo-filament thickness $(H)$, bore diameter $(D)$, and anchorage angle $(A)$ are non-sensitive towards the enhancement of the anchoring performance. 


\subsection{Relation between load-displacement (P-S):}

By removing the undesired values in the experimental groups and averaging experimental results, the $P-S$ load-displacement curves of $L, D, H, R, S$, and $A$ can be attained (Fig. 14-17). According to the destruction characteristics of the anchoring system, each destruction stage can be distinguished by a certain color in the load-displacement curve.

a. The green area of the load-displacement curve illustrates the deformation stage of the geo-filament. The maximum load at this stage is expressed as $N_{i}$.

b. The yellow area of the load-displacement curve demonstrates the stage where the grouting crack or the pullout appears. The maximum load at this stage is expressed as $N_{j}$.

c. The red area of the load-displacement curves depicts the stage of geo-filament pullout or fracture. The maximum load at this stage is expressed as $N_{k}$.

(1) Influence of anchoring length $L$ on the load-displacement curve.

The L-P-S curve (Fig. 13) establishes that an increase in the anchor rod length tends to significantly increase the anchoring force and can effectively control the displacement of the anchoring system under similar pulling force.

When the anchor rod length remains between 800 1500 mm, maximal anchoring force can be increased by increasing the anchor rod length. However, when the anchor rod length is between 1500 3000 mm, increasing the anchor rod length fails to effectively augment the maximal anchoring force. Moreover, when the anchor rod length is about $3000 \mathrm{~mm}$, the maximal anchoring force appears to be extremely close to the fracture tension value of the geo-filament.

(2) Influence of geo-filament thickness $\mathrm{H}$ on the load-displacement curve.

The H-P-S curve is shown in Figure 14. It is evident that the thickness of H2 appears to be double as compared to $\mathrm{H1}$, and the maximal anchoring force only tends to increase by $17.5 \%$. Increasing the geofilament's thickness does not guarantee an effective enhancement in the maximal anchoring force. Moreover, this increase in thickness is also unable to provide an efficient control over the displacement. Therefore, changing $\mathrm{H}$ does not necessarily contribute to the effective improvement of the anchoring performance.

Due to the geometry of the geo-filament sheet, the strength of the soil in the anchorage system appears to be greater than that of the grouting, and an increase in the thickness $(H)$ triggers a certain weakening effect on the integrity of the grouting. Through the experiment, it can be concluded that increasing the thickness of the geo-filament does not enhance the anchorage performance, but rather, it accelerates the crushing of the grouting.

(4) Influence of grouting body strength S on the load displacement curve. 
The S-P-S curve is shown in Figure 15. As the grouting body strength increases, the bonding strength of the grout-rod interface tends to be improved, and the anchoring force increases with the increase in the grouting body strength. Under the same load, the anchoring system's displacement of S1 and S2 appears to be about twice of S3, indicating that the displacement can be effectively controlled by increasing the grouting strength.

(5) Influence of anchor rod surface state R on the load displacement curve.

The R-P-S curve (Fig. 16) illustrates that the maximal anchoring force of the anchor rod RO without a rib is about $4.33 \mathrm{kN}$. The maximal anchoring force of $R 100$ and $R 30$ with a rib is about $7.53 \mathrm{kN}$ and $11.06 \mathrm{kN}$, respectively. The maximal anchoring force increases by $73.90 \%$ and $155.42 \%$ for the respective states after the rib is added. When the tension is greater than $3 \mathrm{kN}$, the displacement of ribbed anchoring system appears considerably lesser than that of the one without a rib. This indicates that the change of geofilament surface state can effectively increase the ultimate anchoring force and control the displacement as well.

(6) Influence of anchoring deployment angle A on the load displacement curve.

The A-P-S curve (Fig. 17) exhibits that the anchoring force seems to undergo a reduction with the increase of the angle, and the deployment also increases under the same load. Since the load applied here does not reach the anchor rod just over a single action line, the anchor is unable to perform optimally. However, keeping in mind construction technology requirements, it is suggested that the layout angle of the anchor rod must be at $10^{\circ}$ for it to perform to its fullest.

\subsection{Strain distribution $(\xi-L)$ characteristics of GF and the grouting interface:}

The analysis of the destruction results of this experiment imply that the anchoring system is ineffective due to the bond-slip present between the geo-filament and the grouting interface, which causes the bolt to pull out.

In the P-S curve (Fig. 13-18), the bond-slip stage is shown as the red segment. The distribution in this stage is similar to that of the brittle failure. The anchoring system exhibits no evident deformation when the anchor rod is suddenly pulled out.

For the anchoring of the raw earth architectural ruins, it appears vital not only to protect the cultural relics while evading the destruction of the grout-soil interface, but also to allow the anchoring system to perform optimally, hence, the anchoring force design needs to have a certain safety reserve.

In accordance to the load displacement curve (P-S), the failure characteristics, and the strain distribution at the geo-filament-grout interface ( $\xi-L$ ), and taking into account the particularity of anchoring of the raw earth architectural ruins, the calculation formula for the design value $N$ of the anchoring force is given as shown in Equation (1) and (2). 
$N_{1}=N_{i}+\left(N_{j}-N_{i}\right) \times 50 \%(1)$

$N_{2}=N_{j}+\left(N_{k}-N_{j}\right) \times 30 \%(2)$

Where $N_{i}$ is the extreme value of the geo-filament deformation stage, with the anchoring system in the elastic stage; $N_{j}$ is the extreme value when the grouting cracks or is pulled out, with the anchoring system in the plastic stage; and $N_{k}$ is the extreme value of the geo-filament pull-out stage, where the anchoring system remains in the bond-slip stage.

The specific values of $N_{i}, N_{j}, N_{k}$ are shown in Tables 5-10. The $N$ values of different experimental lots ( $L$, $H, D, S, R, A)$ can be derived by sorting out the data, as shown in Table 11.

Considering the values of N1 and N2 in Table 11, the strain distribution curves of the geo-filament-grout interface under the action of $N$ of each experimental lot $(L, H, D, R, S, A)$ are extracted, as shown in Figures 19-24. 
Table 11

The $\mathrm{N}$ values in different experimental lots.

\begin{tabular}{|c|c|c|c|c|c|}
\hline Classification & $N$ valt & es $(k N)$ & & & \\
\hline L & $\angle 8$ & $L 12$ & $\angle 15$ & $\angle 20$ & $\angle 30$ \\
\hline$N_{1}$ & 4.23 & 5.03 & 7.45 & 7.18 & 7.45 \\
\hline$N_{2}$ & 7.35 & 8.67 & 13.71 & 14.00 & 14.69 \\
\hline$H$ & $H 1$ & $H 2$ & - & - & - \\
\hline$N_{1}$ & 5.15 & 7.82 & - & - & - \\
\hline$N_{2}$ & 8.88 & 12.26 & - & - & - \\
\hline$D$ & $D 45$ & $D 75$ & $D 85$ & - & - \\
\hline$N_{1}$ & 4.89 & 4.42 & 2.40 & - & - \\
\hline$N_{2}$ & 8.53 & 7.74 & 3.97 & - & - \\
\hline$S$ & S1 & $S 2$ & S3 & - & - \\
\hline$N_{1}$ & 3.35 & 4.82 & 5.96 & - & - \\
\hline$N_{2}$ & 5.24 & 8.75 & 14.78 & - & - \\
\hline$R$ & RO & $R 100$ & $R 30$ & - & - \\
\hline$N_{1}$ & 2.13 & 2.20 & 4.82 & - & - \\
\hline$N_{2}$ & 2.79 & 3.80 & 8.55 & - & - \\
\hline$A$ & $A O$ & $A 10$ & $A 15$ & - & - \\
\hline$N_{1}$ & 4.90 & 4.39 & 3.44 & - & - \\
\hline$N_{2}$ & 8.57 & 7.45 & 5.27 & - & - \\
\hline
\end{tabular}

(1) Design value $\mathrm{N}$ of anchoring force based on the anchor length $\mathrm{L}$.

As evident from Figures 19-24, with the increase of anchoring length L, grout strength S, and geo-filament surface roughness $R$, the anchoring performance of geo-filament appears to be enhanced appreciably, among which, the anchoring length $L$ seems to produce the most striking influence, and the variation of anchoring length $L$ appears to be the most common contributor in practical application.

Figure 19 illustrates that the maximum transmission depth of anchoring force in the geo-filament anchoring system is about $2.2 \mathrm{~m}$, and the stress of the anchor rod suffers significant changes in the central part. Combined with the practical experience and experimental data, the designed anchoring depth of the geo- 
filament bolt should not be more than $2 \mathrm{~m}$, and the optimal anchoring length should be between $1.2 \mathrm{~m}$ and $1.5 \mathrm{~m}$.

By comparing the strain distribution curves of the geo-filament anchor under the action of the design value $N$ of the anchoring force, it seems reasonable to further determine the design value $N$ of anchoring force based on the change of anchoring length L. Considering the safety reserve of the anchoring system and its performance, the calculation formula for $N$ is shown in Equation 3.

$N= \begin{cases}N_{1} & 0<L<1500 \\ N_{2} & 1500 \leq L \leq 2000\end{cases}$

(2) Grouting strength design value.

When the failure of the anchoring system occurs between the grouting and the site soil, the anchoring system performs optimally, and is an ideal location for failure. However, such destruction is detrimental to the site soil as well as non-conducive to the protection of earthen cultural relics. Hence, when designing the anchor rod, it should be given priority that if the damage occurs in the later usage stage, the damaged part appears between the grouting and the anchor rod, to ensure that the site itself is not damaged and remains intact.

The load of the geo-filament bolt is mainly transmitted by the bond stress (shear stress) between the bolt and the grouting body in the anchoring section. The destruction form of the grouting is in the form of shear failure.

By comparing the stress distribution at the interface of the geo-filament-grout in Figures 20-25 and the obtained data, it can be concluded that under the action of the design value of anchoring force $N$, the frontend L/5 segment of the anchor rod experiences about $45 \%$ of the anchoring force, and the damage of the anchorage system begins with the fracture of the end grouting. The impairment of the anchoring system begins with a crack in the front-end grouting of the anchor rod.

Therefore, the stress distribution set of the anchoring system under the action of $N$ can be simply deduced as uniform distribution at the front-end $L / 5$ and triangular distribution at the other parts. The simplified diagram is as shown in Figure 25.

In Figure 25, $\tau_{1}$ is the bonding stress between the geo-filament-grout interface and $\tau_{2}$ is the bonding stress between the grout-soil interface at the bolt front-end L/5 section. According to the balance of internal and external force, $\tau_{1}, \tau_{2}$ can be obtained as:

$\begin{aligned} \tau_{1} & =\frac{9 N}{8 d L} \\ \tau_{2} & =\frac{9 N}{4 \pi D L}\end{aligned}$ 
Where is the width of the geo-filament, and D depicts the bore diameter of grouting (Fig. 7). $\tau_{1}=\frac{\Pi D}{2 d} \tau_{2}$ can be obtained from Equations (4) and (5), $\alpha=\frac{\Pi D}{2 d}$ is defined as the shear stress diffusion coefficient. The relationship of $\tau_{1}$ and $\tau_{2}$ can be written as:

$\tau_{2}=\frac{1}{\alpha} \tau_{1}$

Where we need to satisfy $\tau_{2} \leqslant \frac{\tau_{2}^{\max }}{n}$, and is the safety factor. Therefore, the relation between $\{\backslash$ tau _ 1$\}$ and $\{\backslash$ tau _2\} can be further written as:

$\tau_{1} \leq \frac{\alpha}{n} \tau_{2}^{\max }$

(7)

Through comprehensive analysis, it can be concluded that the shear strength of the grouting should not be more than $\backslash$ frac\{ $\backslash$ alpha $\}\{\mathrm{n}\}$ times that of the soil for the design of the geo-filament bolt.

\section{Conclusions}

In reinforcing small sliders for earthen architectural sites, a GFA has its advantages including easiness in installation, due to its relatively small perforation, and effectiveness in deformation control. A GFA employs a full-length bonding anchoring mechanism, of which an under-applied-force simulation reveal a failure process in three phases, namely filament deformation (elastic deformation), grout cracking (plastic deformation) and filament dislodging (adhesive detachment). Its maximum anchoring force is primarily determined by the anchoring length $(L)$. However, improvement of its force transmission mechanism and failure mode could be achieved by adjusting its grouting strength $(S)$ and surface roughness (R).The design value of the anchoring force $(N)$ is determined by the extreme value of pulling force in each phase of its failure process and L. Approximate $45 \%$ of the $N$ is supported by the segment, $1 / 5$ of $L$, near the end of the bolt. The stress distribution at the soil-anchor interface could thus be simplified as a uniform distribution on the $L / 5$ end segment and a triangular distribution on the rest. The shear strength of the grout (S) should not exceed $\backslash$ alpha times of the shear strength of the reinforced soil. In reinforcing raw-earth building sites, a GFA should be applied desirably with a 1.2-1.5m (not exceeding $2 \mathrm{~m}$ ) anchoring depth, no more than one filament and a perforation of a $40-50 \mathrm{~mm}$ diameter. It is also of note that in real-life cultural heritage conservation projects, stronger methods do not necessarily result in better reinforcing performance. The appropriate methods should always be selected and designed according to the mechanical characteristics of specific.

\section{Declarations}

\section{Availability of data and material statement:}

The authors declared that all data generated or analysed during this study are included in this published article and its supplementary information files. 


\section{Competing interests statement:}

The authors declared that they have no conflicts of interest to this work.

\section{Funding:}

This work was supported by the China Postdoctoral Science Foundation (2020M683672XB), the Youth Science and Technology Fund of XAUAT ( ZR18040), Major Special Projects in the Thirteenth Five-Year Plan of China(2017YFC0702405).

\section{Author contributions:}

Wang Yulan contributed to the conception of the study, performed the experiment and wrote the manuscript;

Guo Jian performed the data analyses and wrote the manuscript;

Lei Fan helped perform the analysis with constructive discussions.

\section{Acknowledgements:}

In the preparation of the paper, the authors hold gratitude for Mr. Xi Lin for his suggestions in proofreading and English wording. The authors would also like to express our special thanks to the reviewers for their constructive suggestions.

\section{References}

1. SUN Man-li. Research status and development of the conservation of earthen sites. Science of Conservation and Archeology, 2007, 19(4): 64-69.

2. ZHANG Jing-ke, GUO Qing-lin, LI Zui-xiong, et al. Preliminary study on anchorage mechanism of earthen sites[M]. Lanzhou: Lanzhou University Press, 2014.

3. BENMOKRANE B, ZHANG B, CHENNOUF A. Tensive properties and pullout behaviour of AFRP and CFRP rods for grouted anchor applications. Construction and Building Materials, 2000, 14: 157-170.

4. RONG Yan, LI Yu-hu, CAO Jing, et al. Application of bakelite rod anchor in protection of Tang Hanguangmen entrance remains[J]. Journal of Shaanxi Normal University (Natural Science Edition), 2016, 44(3):69-74.

5. Zhang J K, Chen W W, Li Z X, Guo Z Q, Wang N. Analysis of in-situ anchoring characteristics of composite anchor containing steel bar $(\Phi 22 \mathrm{~mm})[\mathrm{J}]$. Rock and Soil Mechanics, 2014, 35(11).3139-3147.

6. Xue $Q F$, Jin $X B$, Chen $Y N$, Yang $X H$, Jia $X$, Zhou $Y K$. The historical process of the masonry city walls construction in China during ${ }^{1 s t}$ to 17 th centuries AD[J]. Plos One, 2019, 14(3): 1-16.

7. Li J F, Zhang J K, Wang Nan, Zhao L Y, Guo Q L. Physical model test on working mechanisms of an anchor group system with four wood bolts in rammed earthen sites[J]. Chinese Journal of Rock Mechanics and Engineering, 2019, 38(11): 2321-2331. 
8. Cui K, Wang D H, Chen W W, Ren X F, Liu J, Yang G. Comparative study of anchorage performance of three types of bolts fully grouted by modified glutinous rice mortar[J]. Rock and Soil Mechanics, 2018, 39(2): 498-506.

9. LIU Guo-qing, XIAO Ming, CHEN Jun-tao, et al. Stress analysis method of fully grouted rock bolt in underground caverns[J]. Journal of Huazhong University of Science and Technology (Natural Science Edition), 2017, 45(6):113-119.

10. LU Wei, ZHAO Dong, LI Dong-bo, et al. Study on the force transfer process of the anchorage interface of bamboo bolt in the rammed earth sites[J]. Chinese Journal of Theoretical and Applied Mechanics, 2019, 51(2): 524-539.

11. ZHANG Jing-ke, CHEN Wen-wu, LI Zui-xiong, et al.Field tests on anchorage mechanism of wood bolts for conservation of earthen sites. Chinese Journal of Geotechnical Engineering, 2013, 35(6): 1166-1171.

12. Lu W, Zhao D, Mao XF, et al. Experimental study on bond-slip behavior of bamboo bolt-modified slurry interface under pull-out load.Advances in Civil Engineering, 2018.01: 1-23.

13. Wang Yulan, Dong Zhao, Wei Lu, and Lei Fan. Experimental Research on Destruction Mode and Anchoring Performance of Carbon Fiber Phyllostachys pubescens Anchor Rod with Different Forms. Advances in Civil Engineering, 2018.03: 1-13.

14. Zhang J K, Shan T T, Wang Y C, Wang N, Meng F, Zhao L Y. Mechanical properties of the soil-slurry $(C G N+C)$ interface of anchorage system in earthen sites[J]. Rock and Soil Mechanics, 2019, 40(3): 903912.

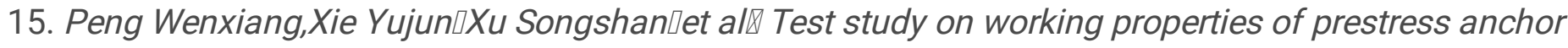

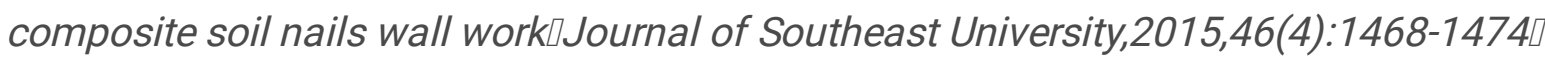

16. H. C. Biscaia, C. Chastre, and M. A. G. Silva, "Linear and nonlinear analysis of bond-slip models for interfaces between FRP composites and concrete," Composites Part B: Engineering, vol. 45, no. 1, pp. 1554-1568, 2013.

17. Chen Y. Experimental study and stress analysis of rock bolt anchorage performance. J Rock Mech Geotech Eng. 2014, 6:428-437

18. L. B. Martin, M. Tijani, and F. Hadj-Hassen, "A new analytical solution to the mechanical behavior of fully grouted rock bolts subjected to pull-out tests," Construction and Building Materials, vol. 25, no. 2, pp. 749-255, 2011.

19. RENFF, YANGZJ, CHENJF, et al. An analytical analysis of the full-range behaviour of grouted rockbolts based on a tri-linear bond-slip model. Construction and Building Materials, 2010, 24: 361-370

20. Nie W, Zhao Z Y, Guo W, Shang J, Wu C. Bond-slip modeling of a CMC rock bolt element using 2D-DDA method[J]. Tunnelling and Underground Space Technology, 2019, 85, 340-353.

21. Wang Z W, Zhao J C, Liu T B. Bond-slip model for horizontal reinforcing bars in reinforced brick masonry[J]. Engineering Structure, 2019, 201: 109770.

22. WU Zhi-min, YANG Shu-tong, ZHENG Jian-jun, et al. Analytical solution for the pull-out response of FRP rods embedded in steel tubers filled with cement grout. Material and Structures, 2010, 43: 597-609 
23. LU Wei, ZHAO Dong, LI Dong-bo, et al. Study on the force transfer process of the anchorage interface of bamboo bolt in the rammed earth sites[J]. Chinese Journal of Theoretical and Applied Mechanics, 2019, 51(2): 524-539.

24. CUI Kai, HUANG Jing-jing, CHEN Wen-wu, et al. Researches on selection of anchor slurry and performance mixed quick lime in earthen ruins[J]. Rock and Soil Mechanics, 2019, 40(6): 2183-2191.

\section{Figures}

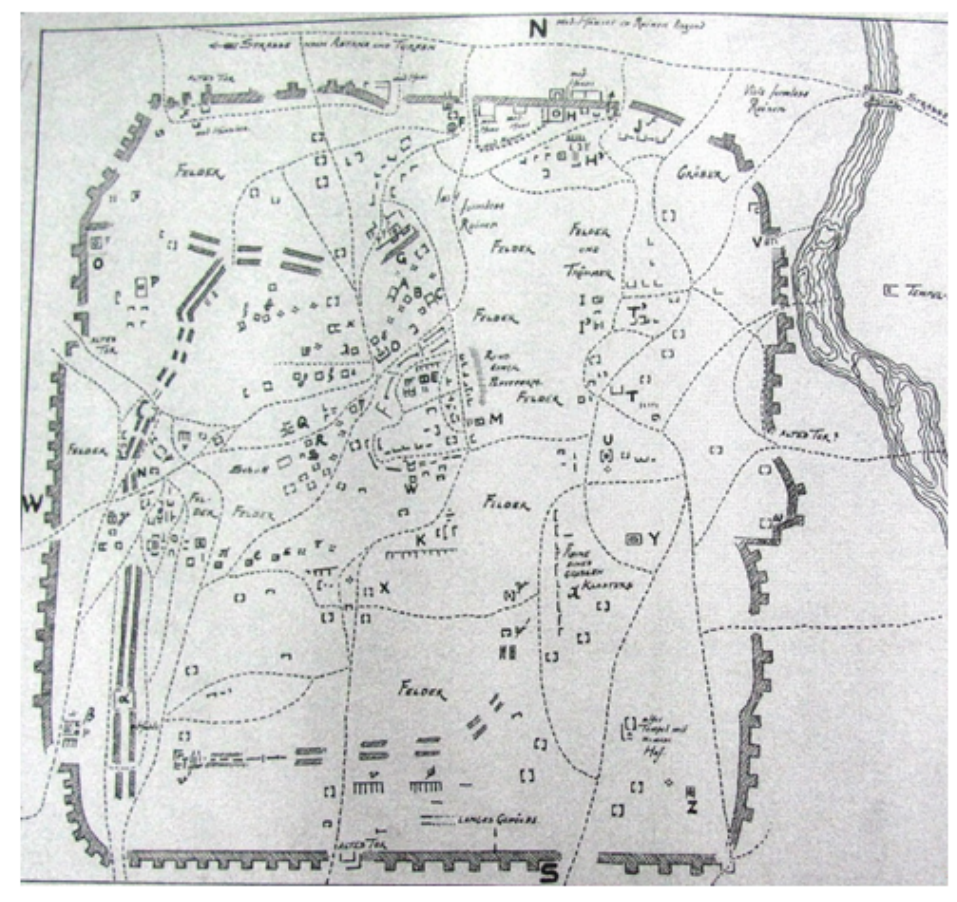

(a) Marc Aurel Stein 's hand drawing(1907).

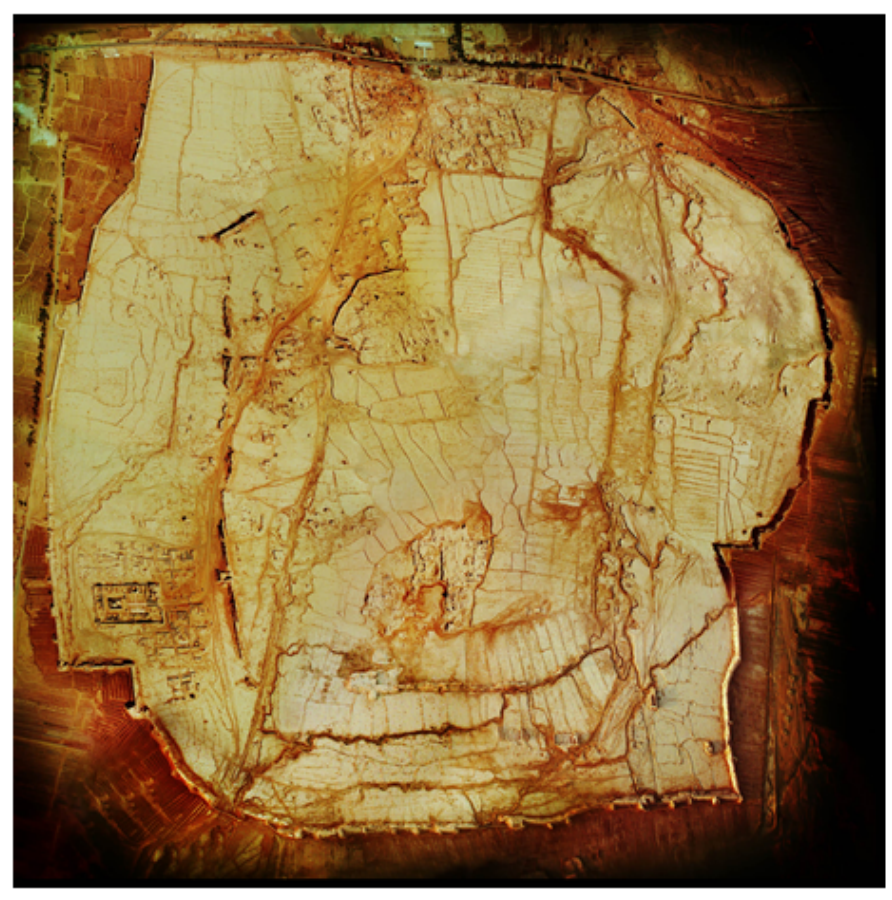

(b) Vertical photograph.

\section{Figure 1}

The plane layout of the Gaochang ruins.

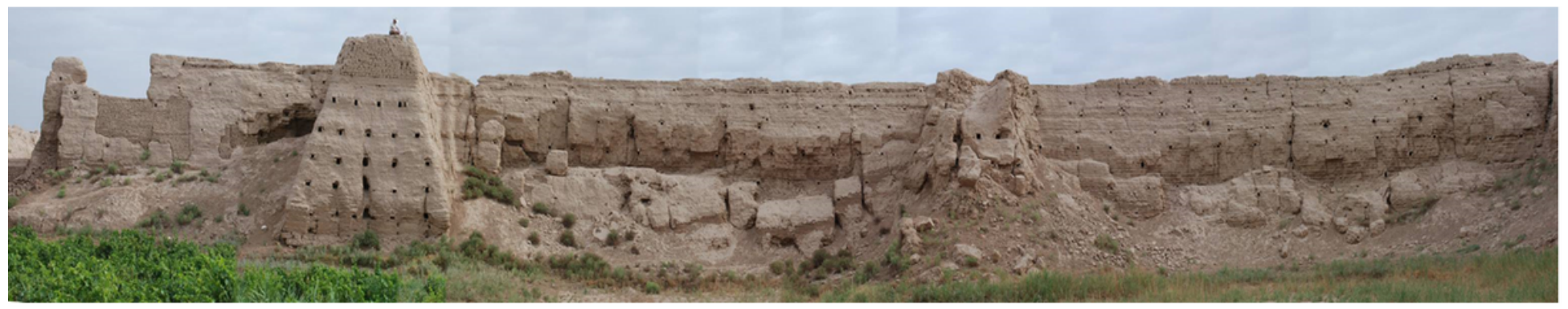

Figure 2

The partial status of outer rampart relics of Gaochang. 

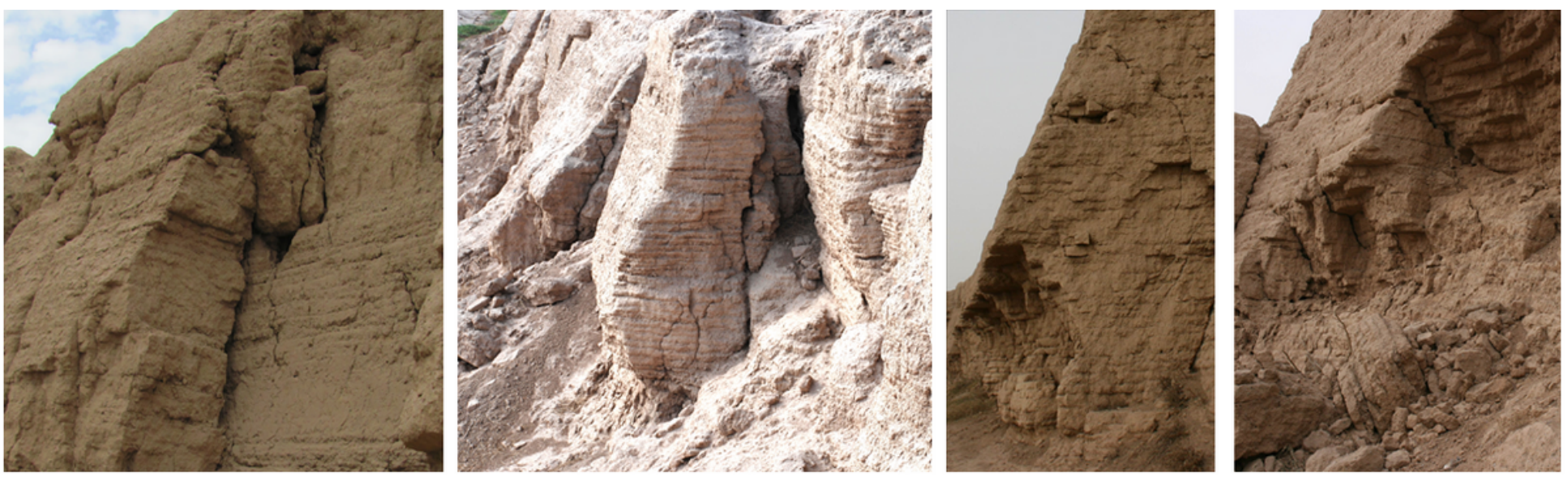

\section{Figure 3}

The hidden structural dangers of the rampart relics.
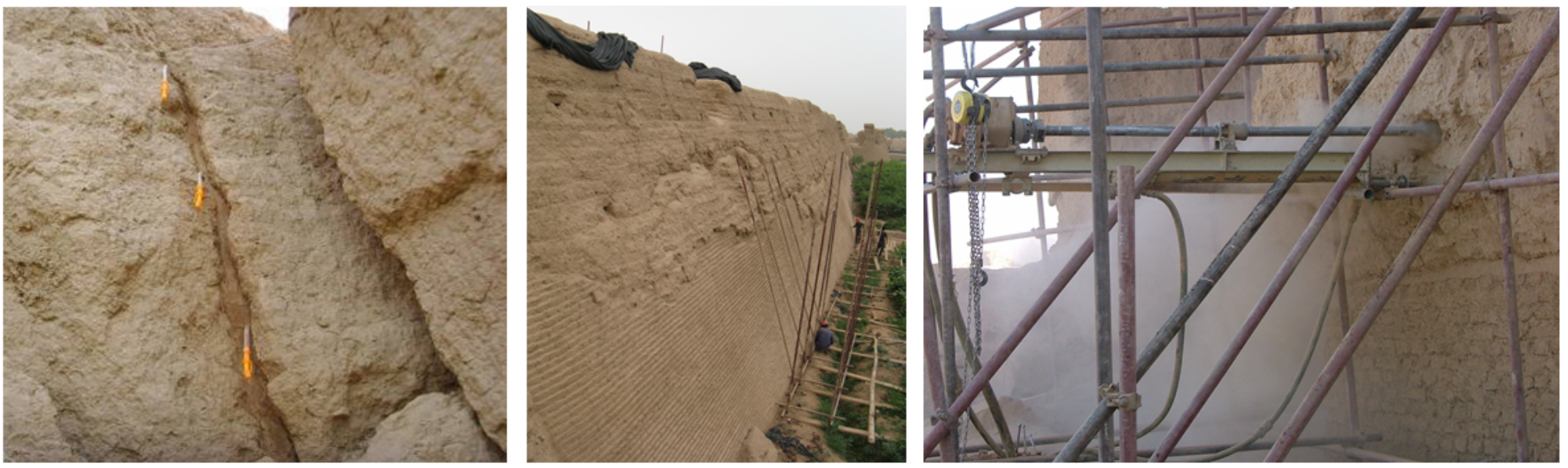

\section{Figure 4}

Grouting, adobe support, bamboo and wood anchor reinforcement.

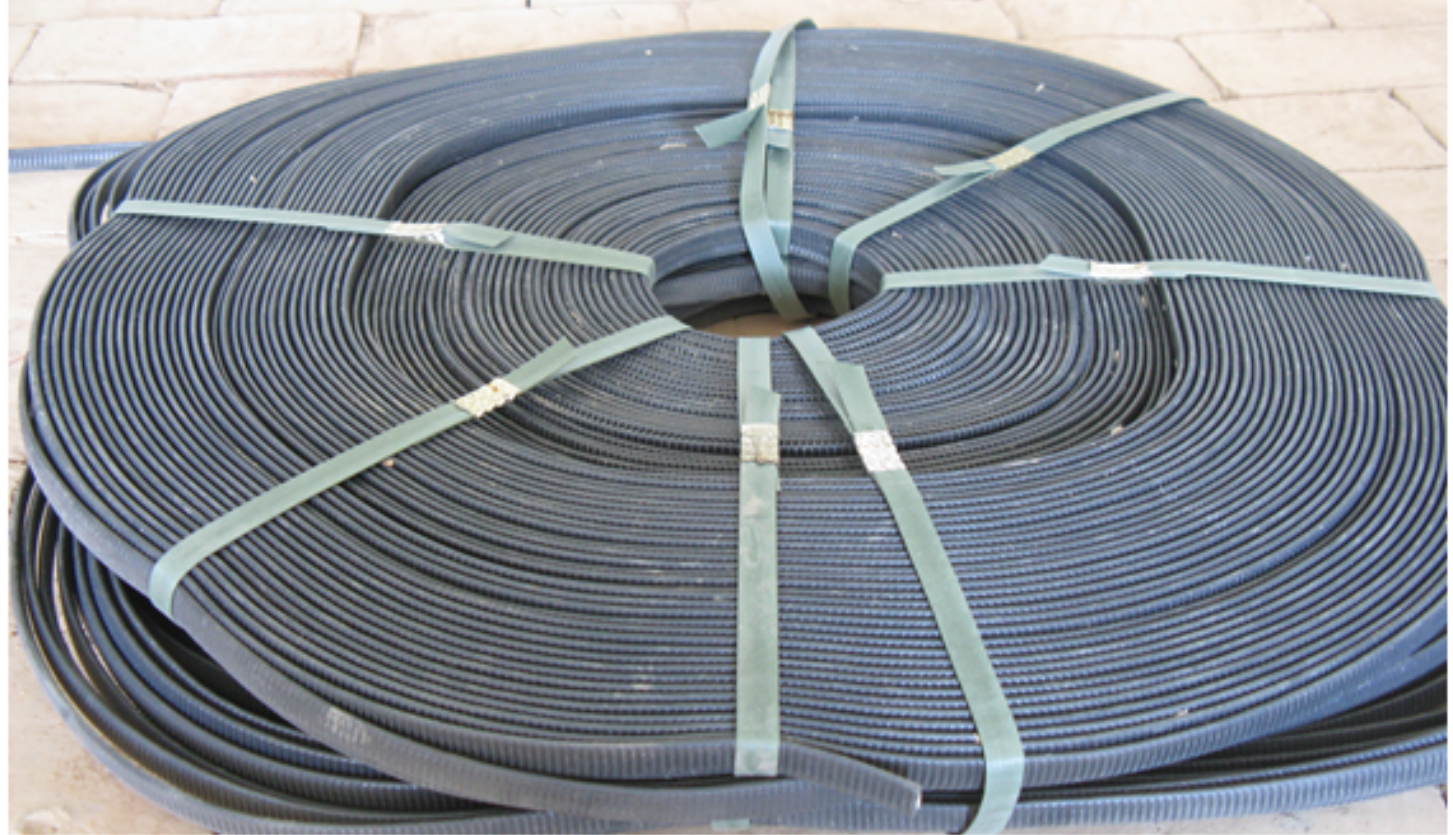


Figure 5

Geo-filament

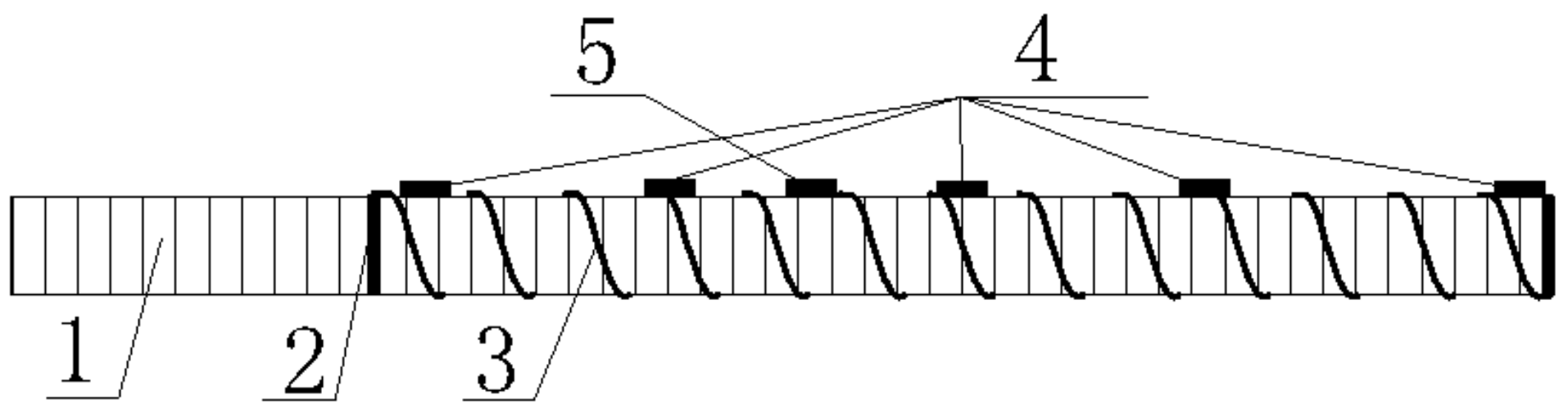

Figure 6

The experiment anchor rod diagram. 1. geo-filament $₫ 2$. three times of hemp rope $₫ 3$. hemp rope $₫ 4$. strain foils $₫ 5$. compensator

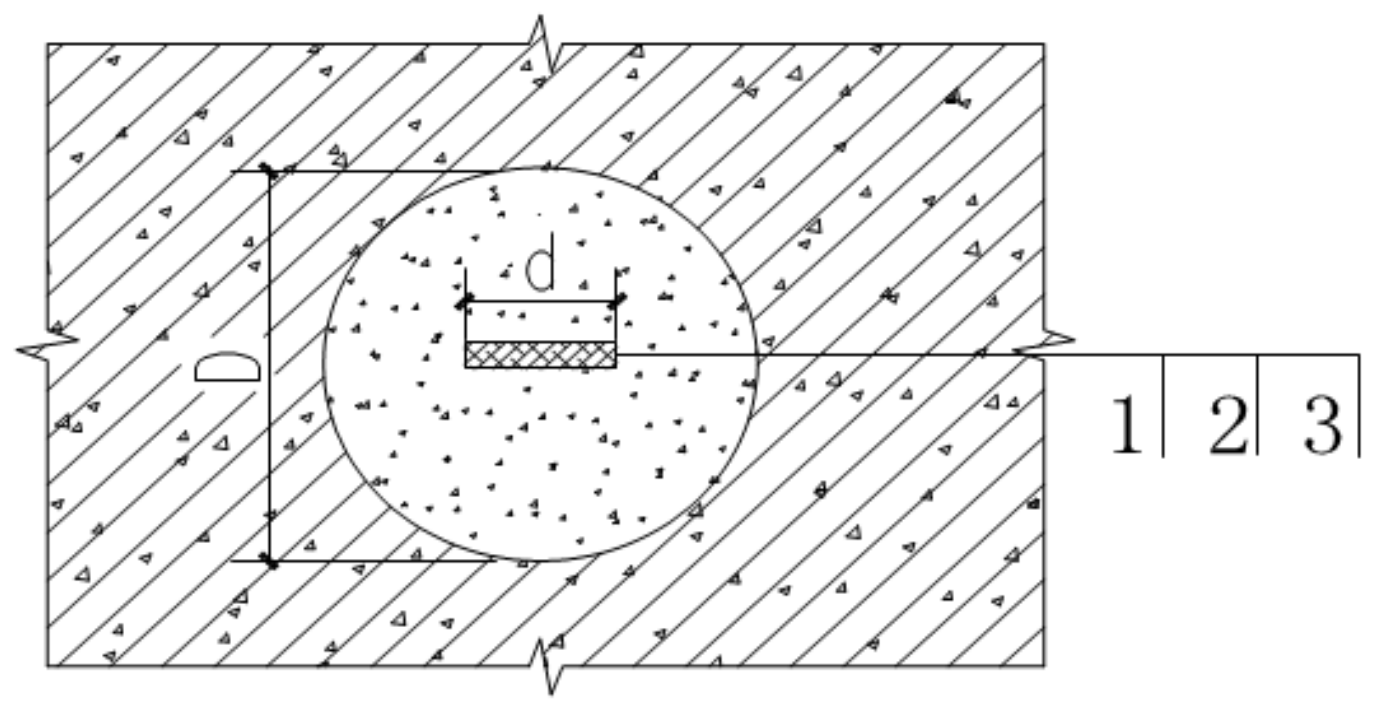

Figure 7

Diagram of the cross section of the anchor. 1. geo-filament; 2. grouting; 3.site soil 

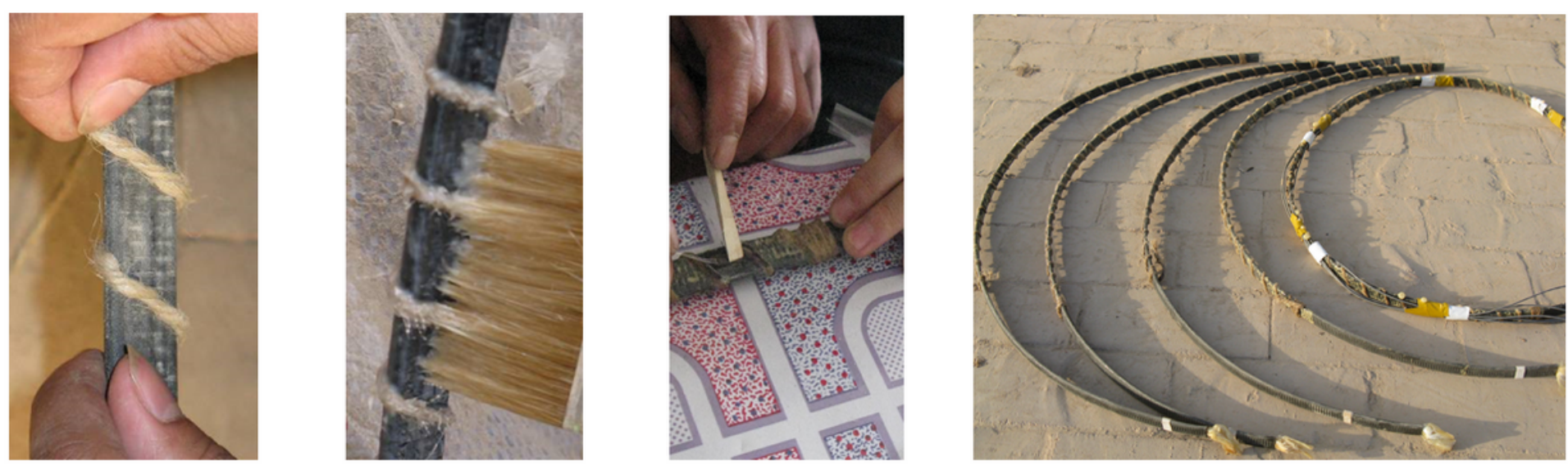

\section{Figure 8}

The manufacture of experiment anchor rod.

\section{Image not available with this version}

\section{Figure 9}

The field anchoring test parts are shown in Figure.

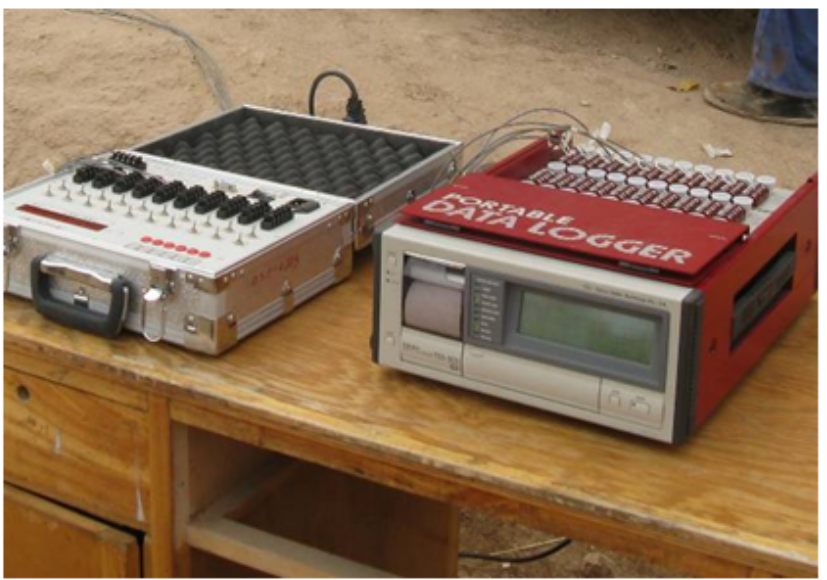

(a) The TDS-303 data collection instrument.

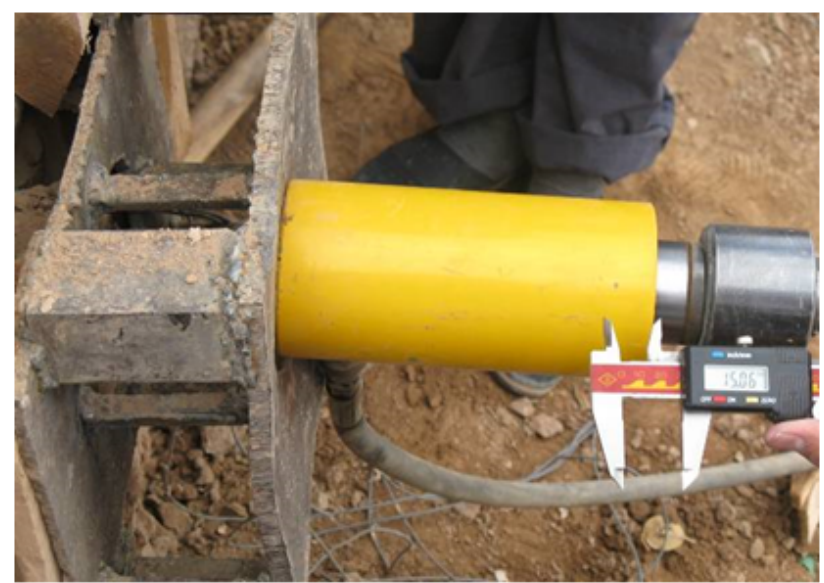

(b) Pull out apparatus and the reaction frame. 


\section{Figure 10}

Test apparatus.

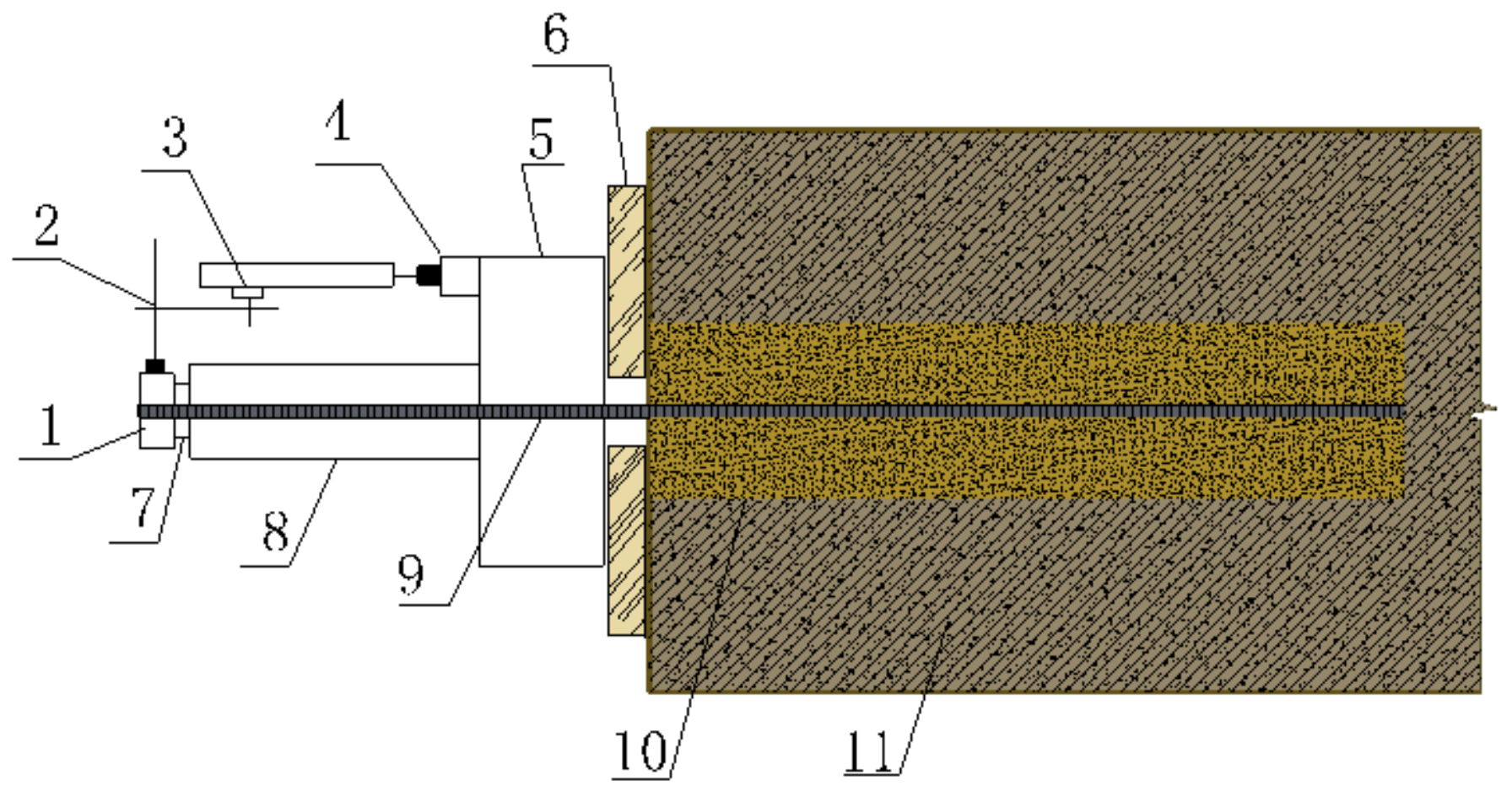

Figure 11

The experimental device diagram.

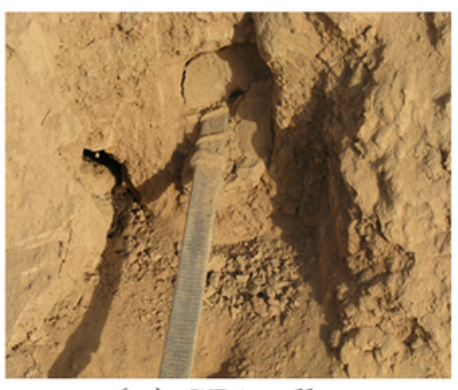

(a) GFA pull out

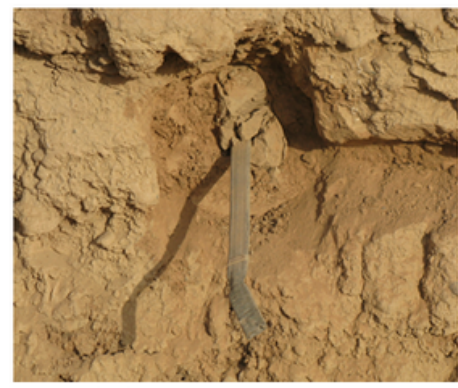

(b) grout-soil damage

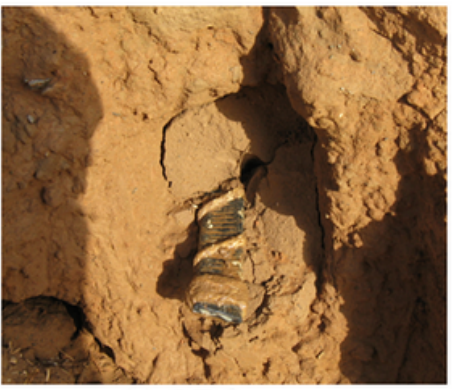

(c) GF fracture

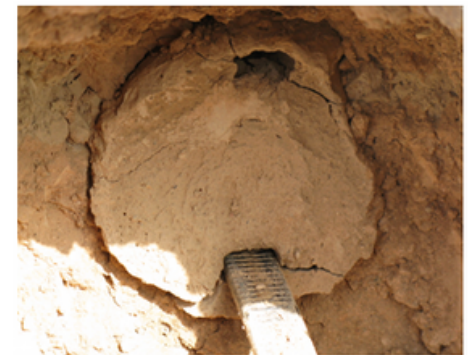

(d) soil-part loosening and grouting pulled out

Figure 12

The main destruction mode. 


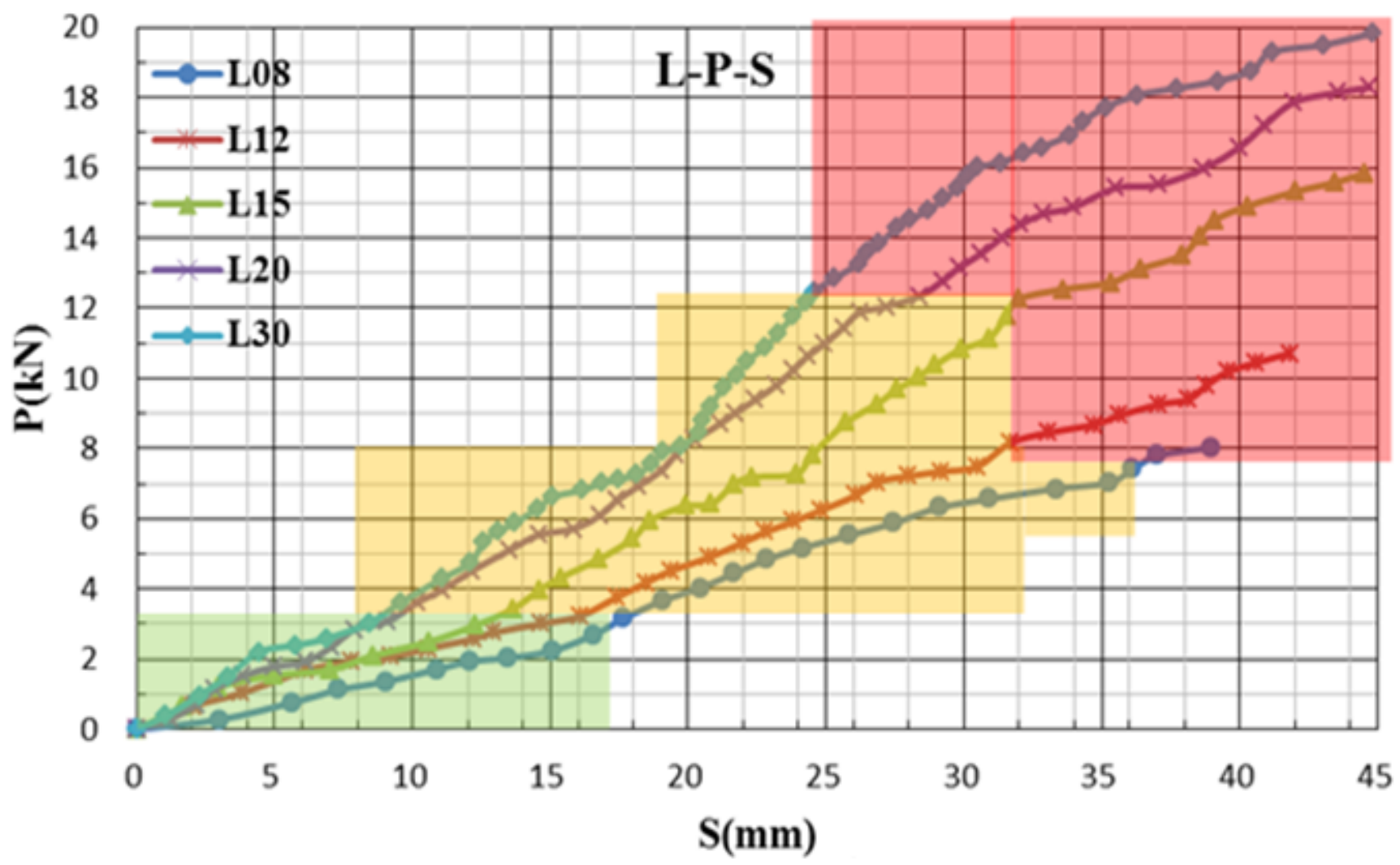

Figure 13

The curve of L-P-S.

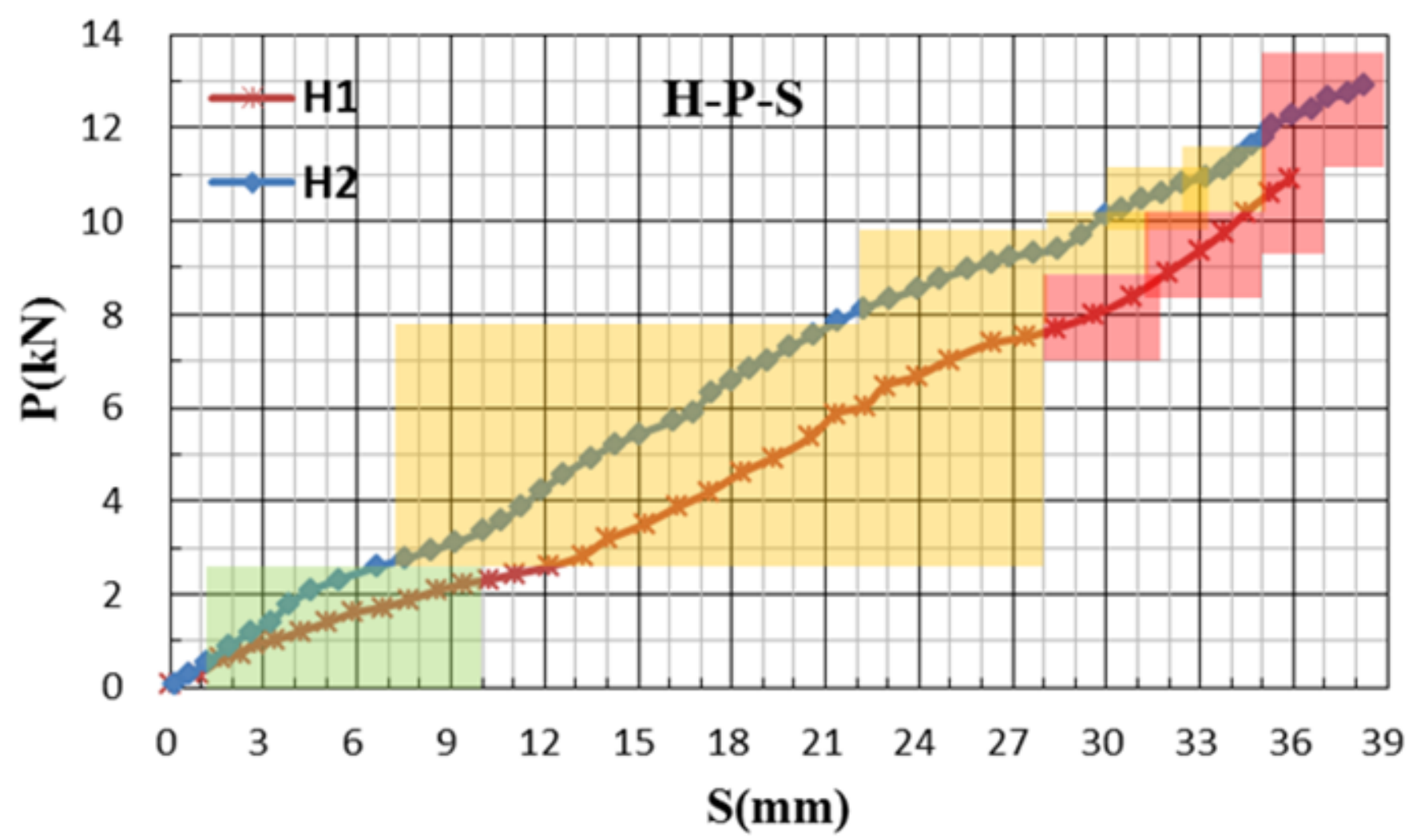

Figure 14 
The curve of H-P-S.

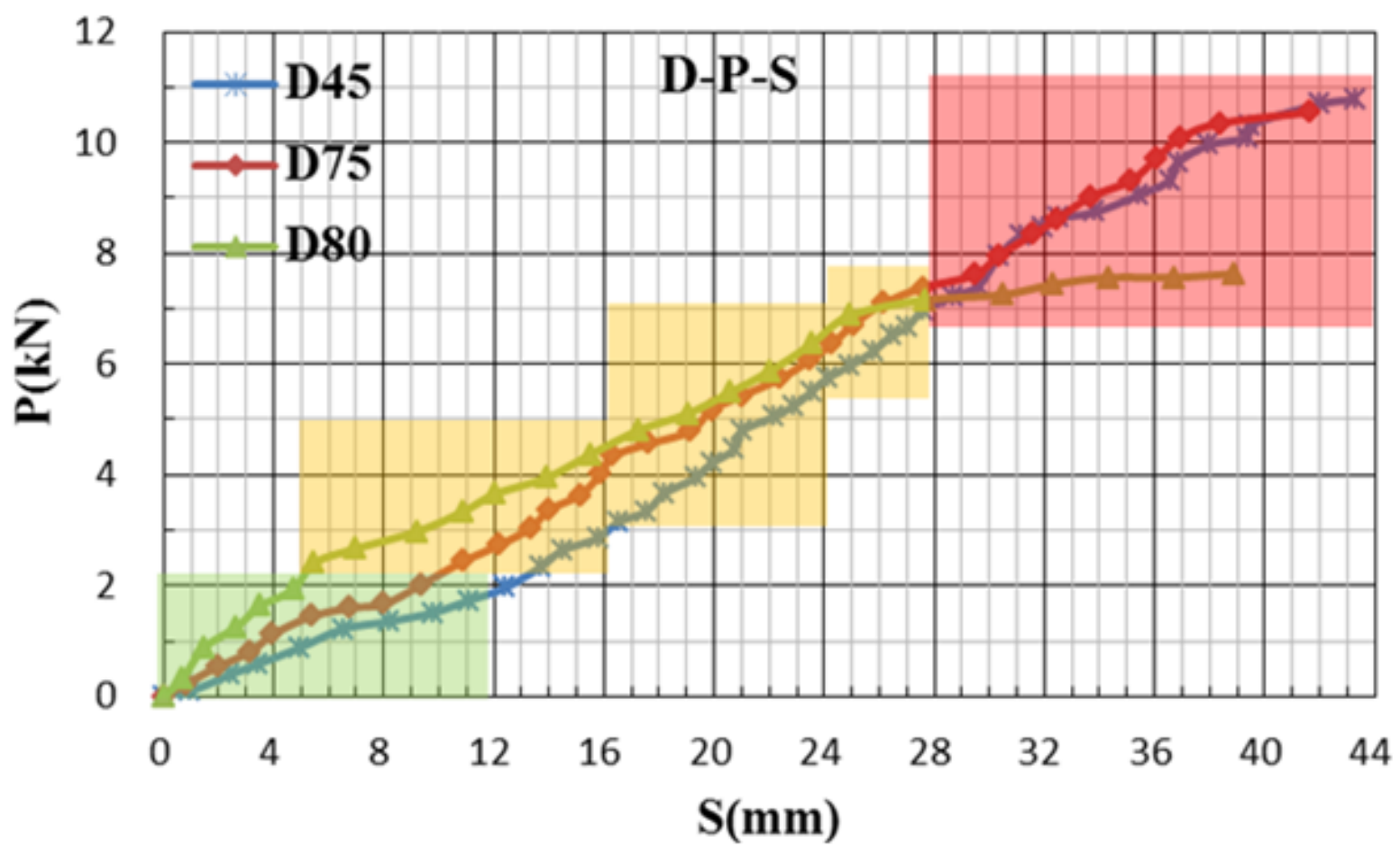

Figure 15

The curve of D-P-S.

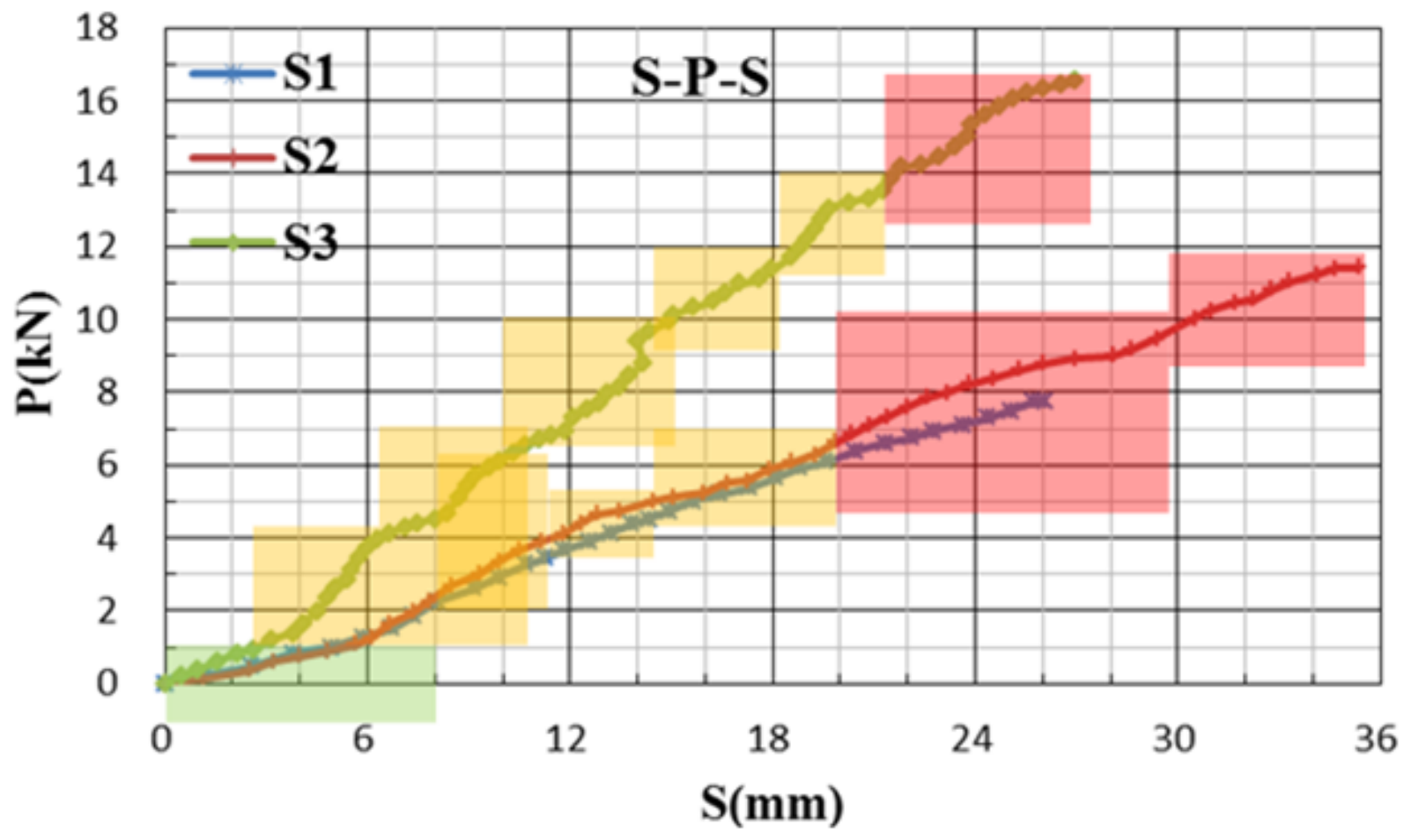

Figure 16

The curve of S-P-S. 


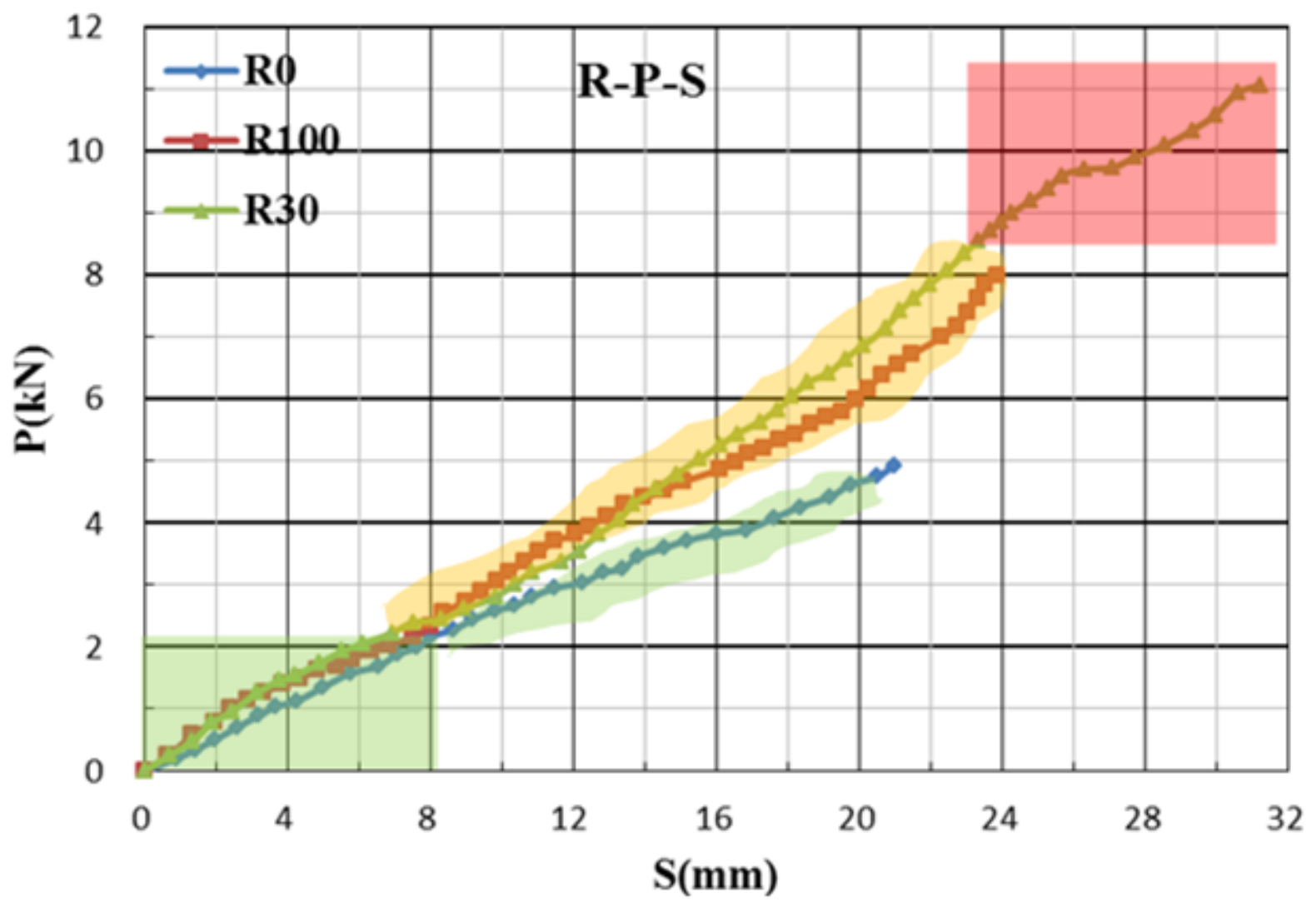

Figure 17

The curve of R-P-S.

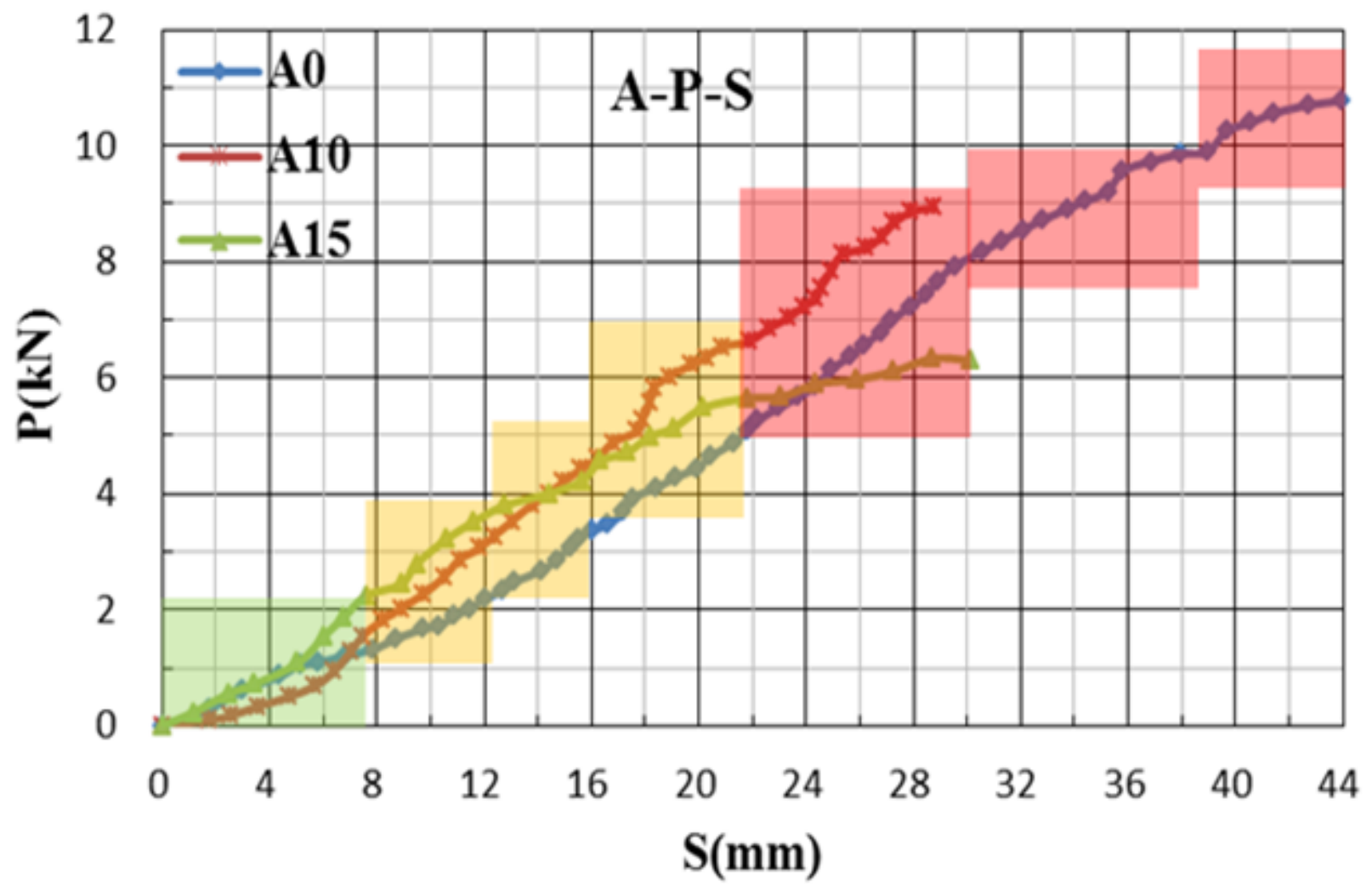

Figure 18 
The curve of A-P-S.

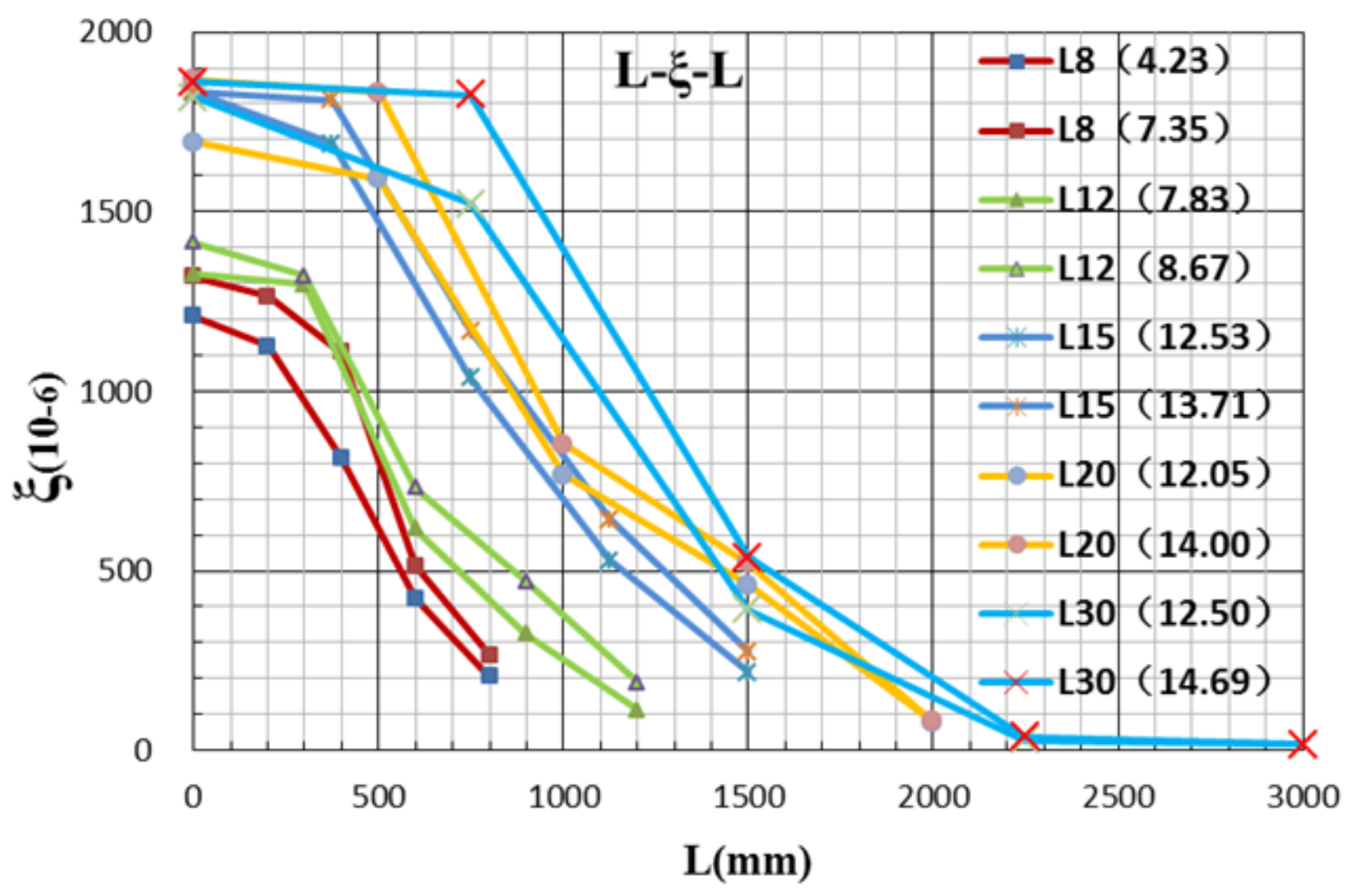

Figure 19

The curve of $L-\xi-L$.

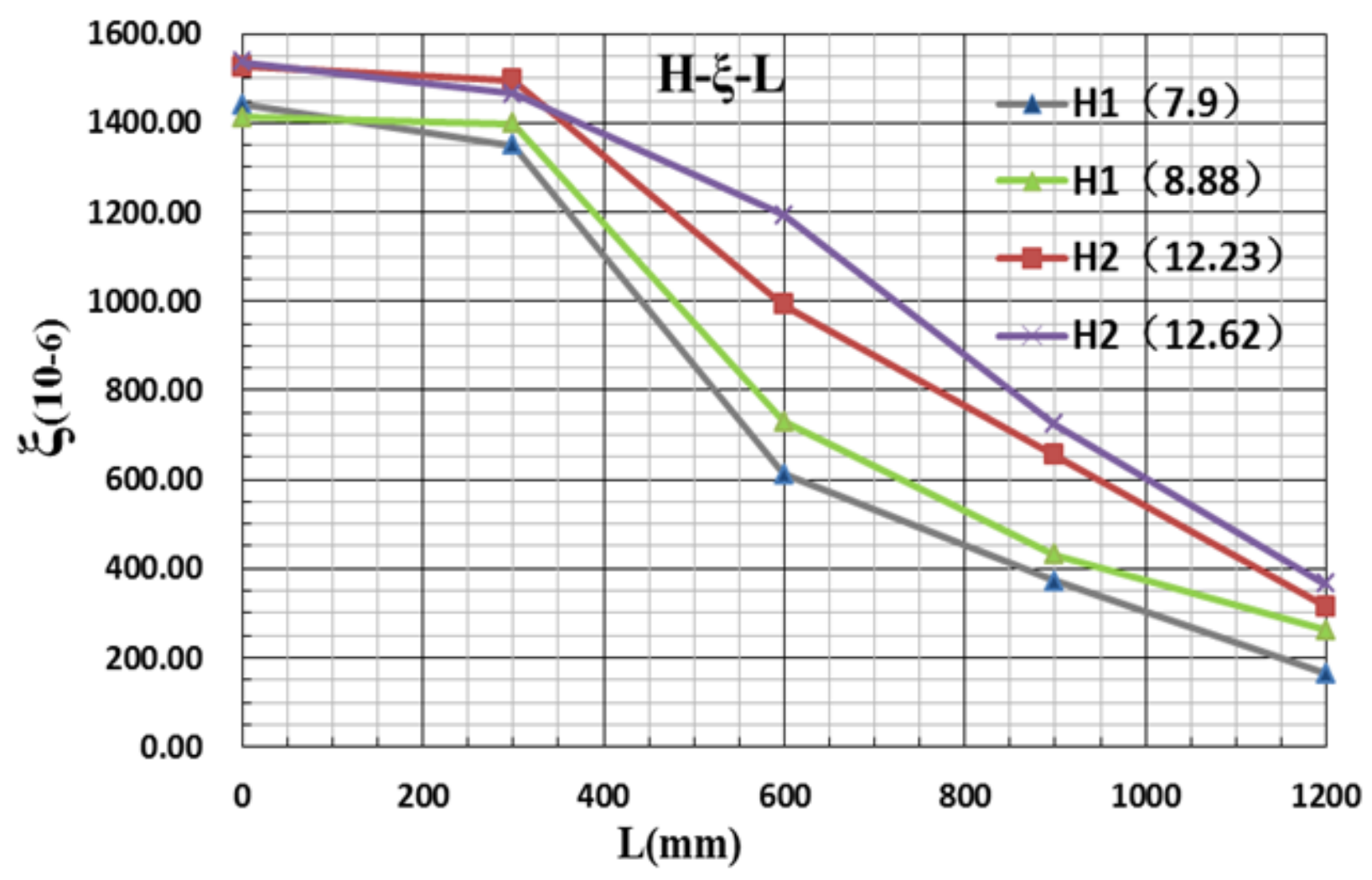


Figure 20

The curve of $\mathrm{H}-\xi-\mathrm{L}$.

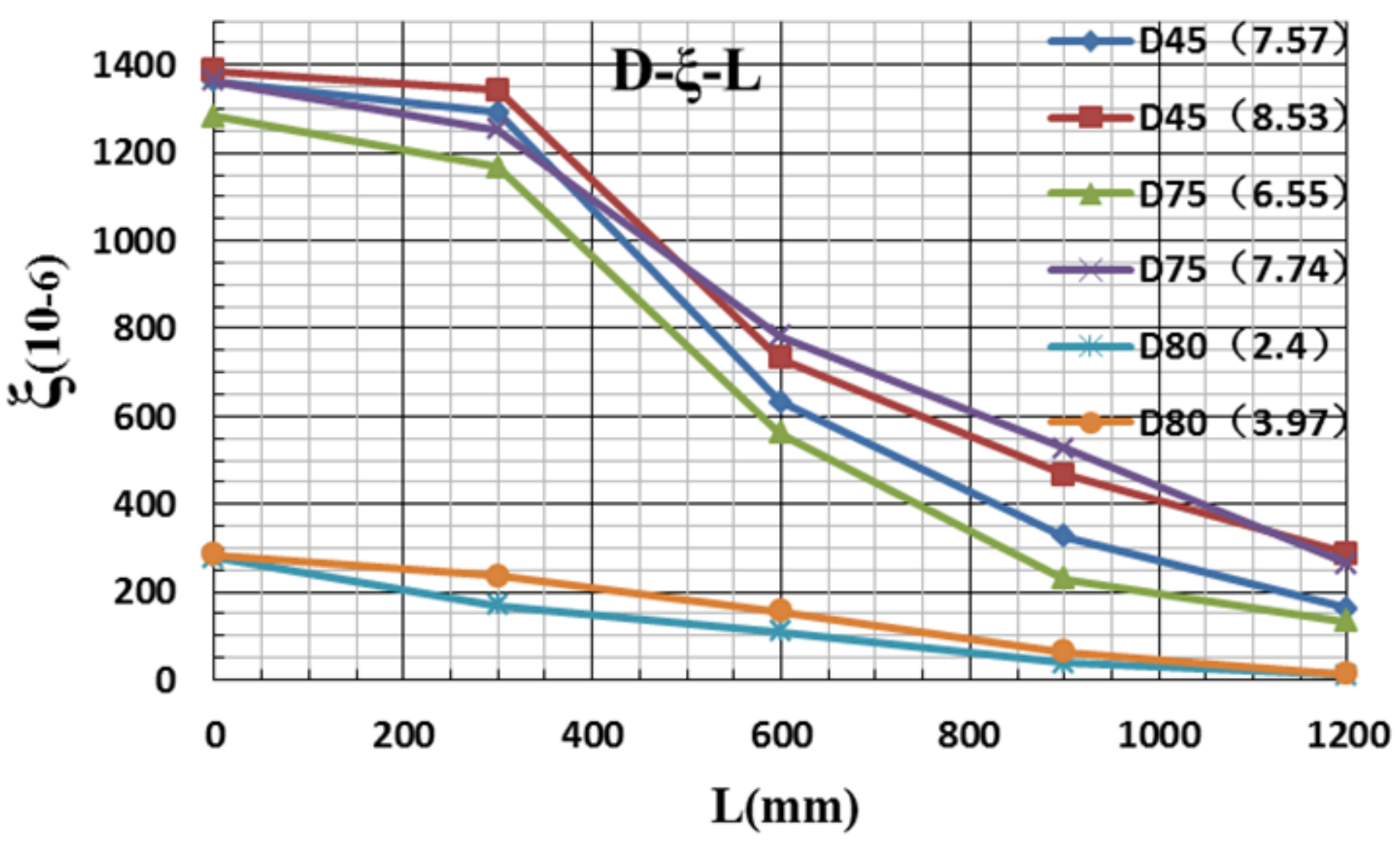

Figure 21

The curve of $D-\xi-L$. 


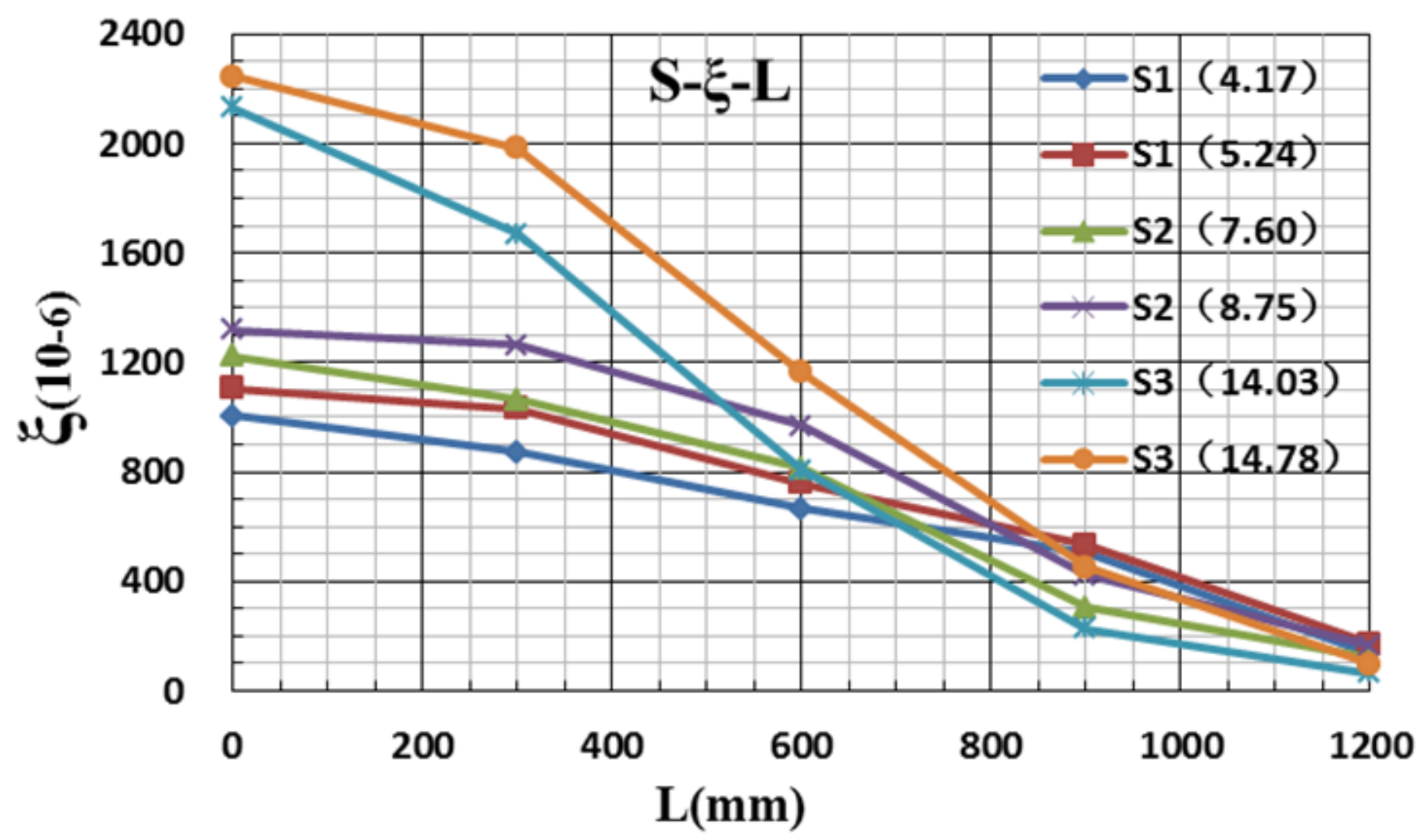

Figure 22

The curve of $S-\xi-L$.

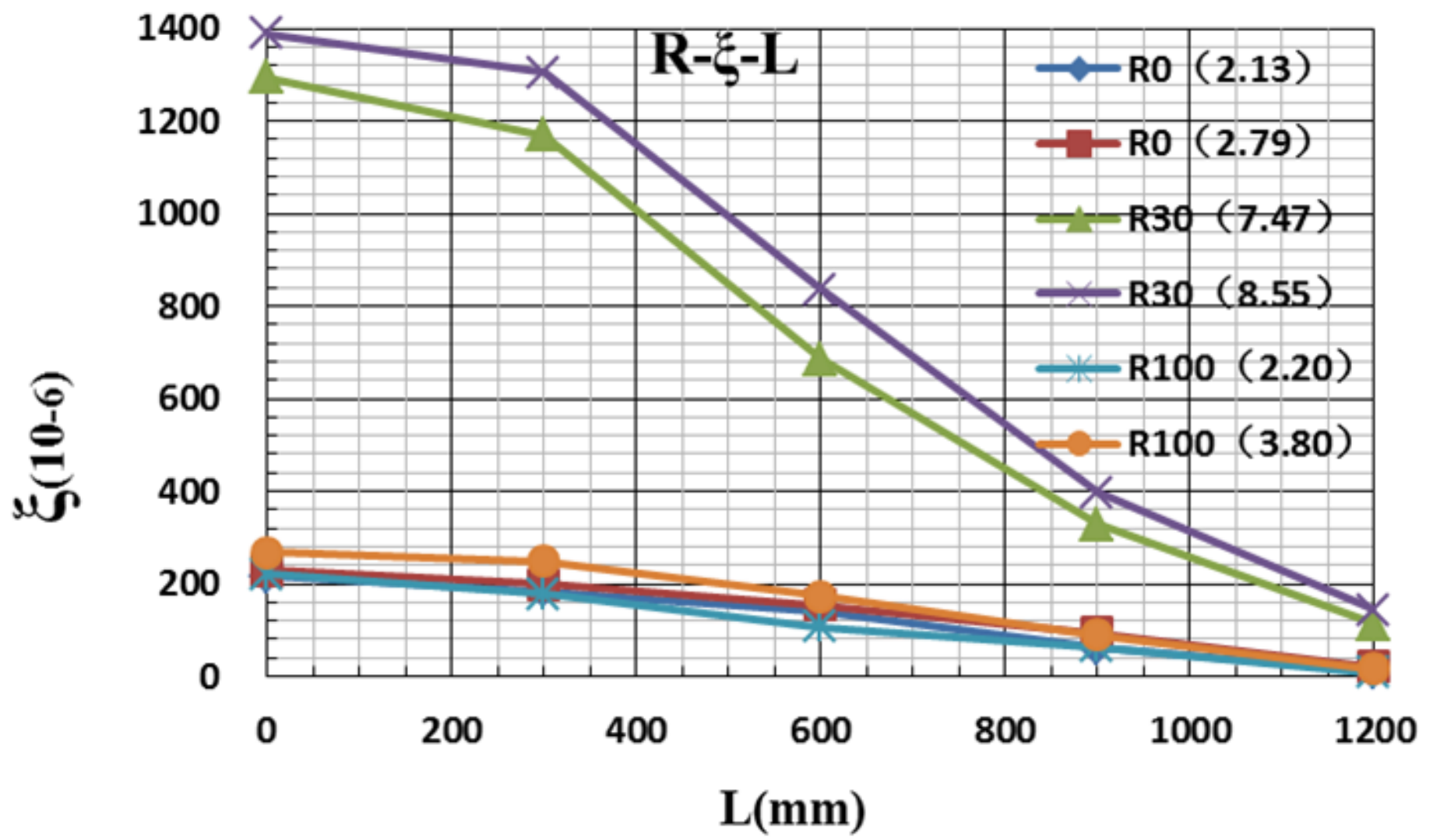


Figure 23

The curve of $R-\xi-L$.

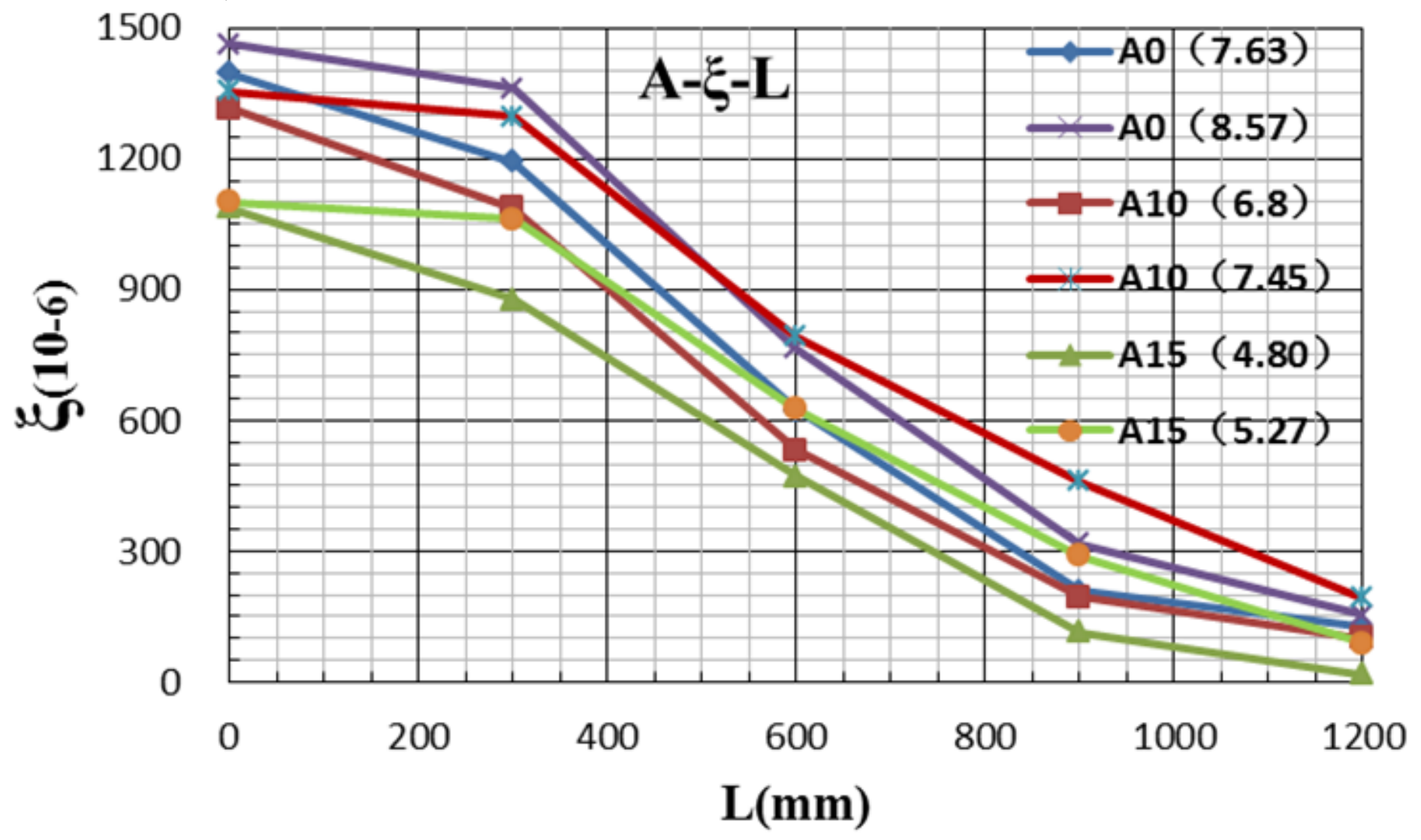

Figure 24

The curve of $A-\xi-L$. 


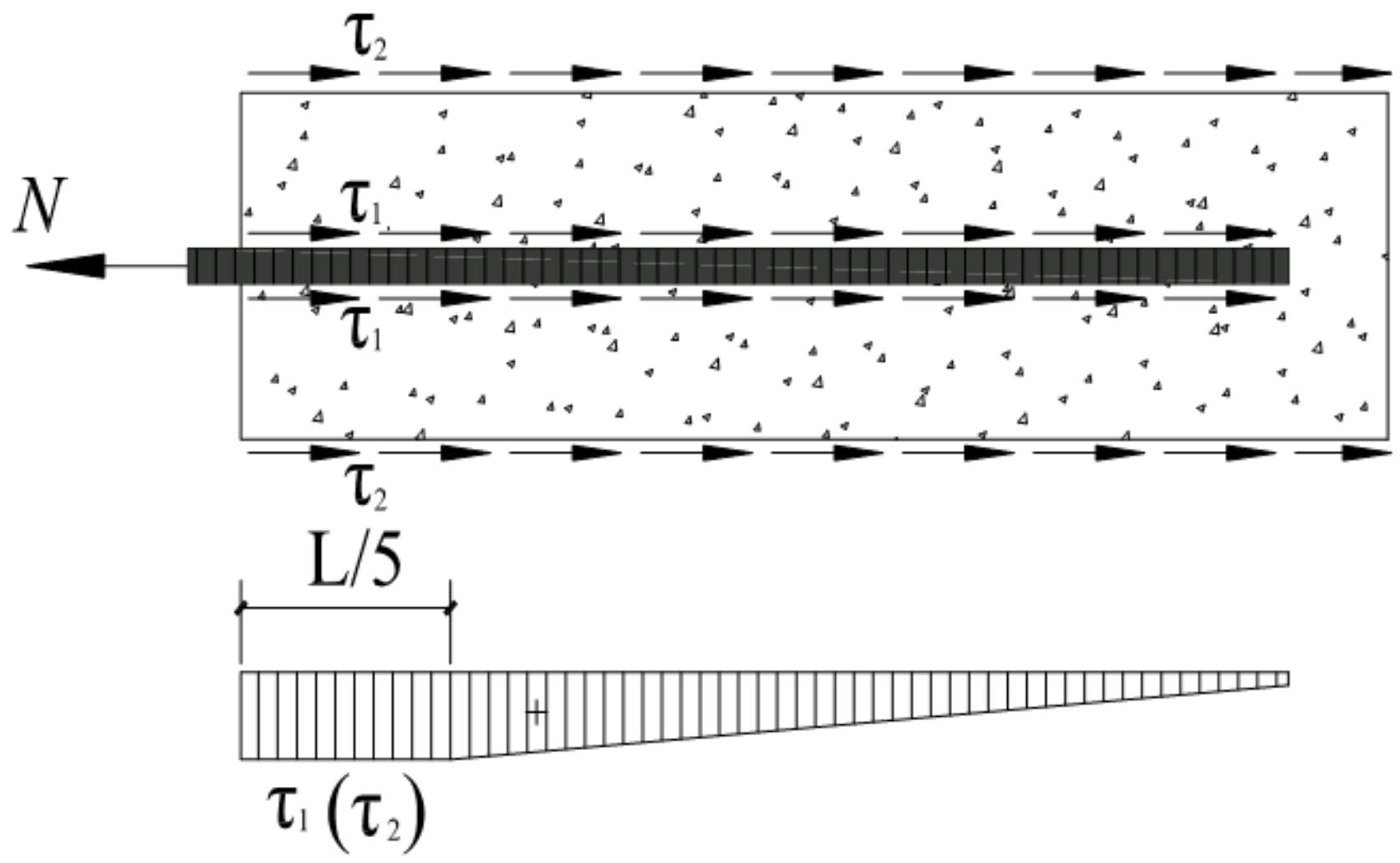

Figure 25

Interface bond stress distribution diagram of GFA under $\mathrm{N}$ action.

\section{Supplementary Files}

This is a list of supplementary files associated with this preprint. Click to download.

- data.xlsx 